\title{
Decay times of transitionally dense specularly reflecting meteor trails and potential chemical impact on trail lifetimes
}

\author{
Wayne K. Hocking ${ }^{1}$, Reynold E. Silber ${ }^{2}$, John M. C. Plane ${ }^{3}$, Wuhu Feng ${ }^{3}$, and Marcial Garbanzo-Salas ${ }^{4}$ \\ ${ }^{1}$ Department of Physics and Astronomy, University of Western Ontario, London, Ontario, N6A 3K7, Canada \\ ${ }^{2}$ Department of Earth Sciences, University of Western Ontario, London, Ontario, N6A 3B7, Canada \\ ${ }^{3}$ School of Chemistry, University of Leeds, Leeds, LS2 9JT, UK \\ ${ }^{4}$ University of Costa Rica, San Jose, Costa Rica \\ Correspondence to: Wayne K. Hocking (whocking@uwo.ca)
}

Received: 20 August 2016 - Accepted: 31 October 2016 - Published: 2 December 2016

\begin{abstract}
Studies of transitionally dense meteor trails using radars which employ specularly reflecting interferometric techniques are used to show that measurable hightemperature chemistry exists at timescales of a few tenths of a second after the formation of these trails. This is a process which is distinct from the ambient-temperature chemistry that is already known to exist at timescales of tens of seconds and longer in long-lived trails. As a consequence, these transitionally dense trails have smaller lifetimes than might be expected if diffusion were the only mechanism for reducing the mean trail electron density. The process has been studied with four SKiYMET radars at latitudes varying from 10 to $75^{\circ} \mathrm{N}$, over a period of more than 10 years, $24 \mathrm{~h}$ per day. In this paper we present the best parameters to use to represent this behaviour and demonstrate the characteristics of the temporal and latitudinal variability in these parameters. The seasonal, day-night and latitudinal variations correlate reasonably closely with the corresponding variations of ozone in the upper mesosphere. Possible reasons for these effects are discussed, but further investigations of any causative relation are still the subject of ongoing studies.
\end{abstract}

Keywords. Atmospheric composition and structure (middle atmosphere - composition and chemistry)

\section{Introduction}

Radar studies of meteor trails provide valuable information both about the meteors that cause them and the environment in which they ablate. Techniques vary, from studies of specularly scattering trails to head-echo studies and forward-scatter procedures. The most common instruments currently in use are probably backscatter meteor radars, modelled largely on the SKiYMET design (Hocking et al., 2001). These radars are mainly used in conjunction with underdense meteors, and they have been used extensively to study winds and temperatures in the mesopause region.

Initial studies of these underdense trails concentrated on ambipolar diffusion as the main mechanism by which the trails were dissipated, and consequently it was possible to use the relevant theory to determine ambipolar diffusion coefficients and hence temperatures around the mesopause region (Hocking, 1999a, and references therein). However, further research showed that chemistry may also play a role in this trail destruction, particularly at altitudes below $83-84 \mathrm{~km}$ (e.g. Lee et al., 2013, and references therein, although the very high attachment rate of electrons to aerosols used in that paper is open to discussion).

However, these same radars can also be used to study another class of meteor trail. In radar work, the trail density partly determines the nature of the scattering process. The trail density is often defined in terms of its so-called "line density", which is the total number of electrons in the trail (irrespective of distance from the centre line) per unit length of trail. If the line density is less than typically $10^{14}$ electrons $\mathrm{m}^{-1}$, VHF and lower-frequency radio waves can generally penetrate the plasma of the trail. As the trail expands laterally, thereby diminishing its mean electron density, radio-wave penetration becomes even easier. On the other hand, if the line density exceeds typically $10^{16}$ electrons $\mathrm{m}^{-1}$, the plasma density is initially too high for VHF and lower frequency radio waves to penetrate, and 
radio waves which impinge on the "edge" of the trail closest to the radar reflect from the trail as if striking a mirror. However, in time the trail expands and the mean electron density is reduced, eventually allowing penetration of the radio waves.

These latter class of meteor trails, termed "overdense", represent a relatively small, but not negligible, percentage of meteors detected by backscatter meteor radars. For many applications they are ignored, but our purpose here is to focus on them. They have many features that distinguish them from underdense meteors, including different radar backscatter signatures and somewhat higher temperatures, and they may have associated shock fronts, as will be further discussed in the next section. An intermediate class of meteor trails exists which has some characteristics of overdense meteors and (at times) some of underdense ones. These are often referred to as "transitionally overdense" and are taken to have electron line densities between $2.4 \times 10^{14} \mathrm{~m}^{-1}$ and $10^{16} \mathrm{~m}^{-1}$. We will collectively refer to the overdense and transitionally overdense trails as "non-underdense".

These non-underdense echoes may last many seconds if observed with the right radars. Normally they are most evident with forward-scatter radars and less easy to study with backscatter radars. The differences are described in Appendix A. The reason that overdense meteors are seen for longer periods of time with forward-scatter radars relates to radar geometry: as shown in Appendix A, the path lengths for radio-wave rays reflected from the "top" and "bottom" of the trail need to be less than a half-wavelength, and this is achievable at much larger trail widths when using forward-scatter geometry. In backscatter mode, trails disappear as soon as they become both underdense and also exceed a diameter of about half a wavelength (more exact criteria are discussed in Appendix C).

Overdense meteors are often more frequently studied with forward-scatter radars (e.g. Cevolani et al., 1999; Cevolani and Pupillo, 2003). However, forward-scatter radars have very poor height resolution, again because of geometrical considerations.

These long-lived meteor trails seen with forward-scatter radars are known to be depleted by ozone chemistry in their final stages. This process takes place at temperatures close to the ambient temperature, the meteor trail having substantially cooled by this time. This so-called "thermalized" overdense meteor trail chemistry is relatively well understood (e.g. Baggaley, 1978; Plane, 2003). These processes involve meteoric ions and the atmospheric constituents $\mathrm{O}$ and $\mathrm{O}_{3}$, and they have been well investigated as a result of a number of theoretical and laboratory kinetic investigations, in addition to radar and experimental studies of the meteor trail (Plane, 2003; Plane et al., 2015). More precisely, exothermic chemical reactions between ozone (as the controlling neutral mesospheric gas) and meteor metal ions, followed by dissociative recombination, play a primary role in removal of electrons (Reactions R1-R2 below) from the thermalized overdense meteor trains (Baggaley, 1979). These reactions are as follows:

$\mathrm{M}^{+}+\mathrm{O}_{3} \rightarrow \mathrm{MO}^{+}+\mathrm{O}_{2}$,

$\mathrm{MO}^{+}+\mathrm{e} \rightarrow \mathrm{M}+\mathrm{O}$,

$\mathrm{MO}^{+}+\mathrm{O} \rightarrow \mathrm{M}^{+}+\mathrm{O}_{2}$,

where $\mathrm{M}^{+}$is a common meteoric ion (e.g. $\mathrm{Fe}^{+}, \mathrm{Mg}^{+}$). Comprehensive work investigating the $\mathrm{Mg}^{+}$reactions with $\mathrm{O}_{3}, \mathrm{O}_{2}, \mathrm{CO}_{2}$, and $\mathrm{N}_{2} \mathrm{O}$ was conducted by Whalley et al. (2011). The rate coefficient for the reaction $\mathrm{Mg}^{+}+\mathrm{O}_{3} \rightarrow \mathrm{MgO}^{+}+\mathrm{O}_{2}$ corresponds to the Langevin capture rate coefficient and is independent of temperature (Whalley et al., 2011). Reaction (R3), which was studied by Whalley and Plane (2010), competes with dissociative electron recombination (Reaction R2), but only in underdense trails where the ratio of the electron to $\mathrm{O}$ density is lower. The other major meteoric metal ion, $\mathrm{Fe}^{+}$, undergoes similar reactions, and the dissociative recombination reaction has recently been studied for the first time (Bones et al., 2016). The rates of the analogous $\mathrm{Si}^{+}$reactions are also similar to those of $\mathrm{Mg}^{+}$(Plane et al., 2016). $\mathrm{Na}^{+}$is a less abundant meteoric metal ion with a different chemistry: it does not react with $\mathrm{O}_{3}$ and instead clusters with $\mathrm{N}_{2}$ in a pressuredependent three-body reaction (Plane et al., 2015). However, $\mathrm{Na}^{+}$should comprise less than $10 \%$ of the meteoric ions in a relatively dense trail where ablation is likely to have been efficient (Vondrak et al., 2008).

Jones et al. (1990) and Jones and Simek (1995) introduced a technique to allow measurement of ozone densities using the theory introduced by Baggaley (1979) and others, employing forward-scatter lifetimes. Examples were shown by Cevolani et al. (1999) and Cevolani and Pupillo (2003), although, as discussed above, vertical resolution was quite poor.

However, in dealing with backscattered echoes with a specularly reflecting system, we never see meteor trails of the ages that can be measured with forward-scatter radars. Typically our longest-lived echoes are little more than a second or two, while the trail is still quite hot. Hence, any results that we observe with our trails must be considered quite different to the ones observed with forward-scatter radar. Nevertheless, our results will involve ozone, albeit in a different manner.

Since the paper will primarily concern non-underdense meteor trails, Sect. 2 gives an overview of some of the characteristics of transitional and fully overdense meteor trails, including temperatures, and discusses how they are distinct from underdense meteors. Section 3 discusses the different classes of meteor trails observed with a VHF SKiYMET radar. Following that, the locations and characteristics of the radars used in the study are presented in Sect. 4. Extensive description of the analysis then ensues in Sects. 5 and 6, including studies of seasonal and day-night variations. Section 7 discusses some chemistry and possible mechanisms 
for the observed results, including the likely central role of ozone. Conclusions are presented in Sect. 8.

\section{Characteristics of strong and transitionally dense meteor trails}

We will consider trails with half-lives (as seen by a specularly reflecting radar) of up to $2 \mathrm{~s}$, as this is the cut-off scale generally used with SKiYMET radars. Tests using a similar radar, but without a restriction of the timescale, showed that longer-lived meteors were very rare (typically 1 out of 2000 detections), so the number of "missed" meteors should be very small. (Note that we use half-lives here, as is normal for meteor decay times: later, in discussing chemistry, we will consider $e$-folding times, which is more common in chemistry; the ratio of half-life to $e$-folding lifetime is $\ln 2$.)

As noted, meteors with trail line densities in excess of $10^{16}$ electrons $\mathrm{m}^{-1}$ are defined as overdense, though the demarkation density of $10^{16} \mathrm{~m}^{-1}$ is a little arbitrary. Meteor radars with a frequency below a few hundred megahertz will observe particular characteristics which identify the echoes as overdense. While underdense echoes generally show a broadly exponential decay in amplitude with time, usually lasting less than about $0.2 \mathrm{~s}$, overdense echoes have a non-exponential decay of the trail echo strength, and often demonstrate an approximately flat backscattered signal strength for a few tenths of a second or more before rapid signal loss occurs. Their lifetimes are usually in excess of a few tenths of a second. Reasons are discussed in Appendix A, and later in Appendix C.

A second line-density scale often invoked in meteor trail studies is the "transitional" density, which is the density which is considered as an upper limit for underdense meteors. This corresponds to a line density of about $2.4 \times 10^{14}$ electrons m$^{-1}$ (McKinley, 1961; Poulter and Baggaley, 1977, 1978). Trails with line densities between $2.4 \times 10^{14}$ and $10^{16}$ electrons $\mathrm{m}^{-1}$ are considered as transitional, as already discussed. Studies suggest that the overdense trails are produced by particles with diameters between approximately 4 and $20 \mathrm{~mm}$, having visual magnitudes $0 \leq M_{\mathrm{v}} \leq 4.4$ (the latter value corresponding to the visual magnitude boundary for a small fireball) (e.g. Sugar, 1964).

Such divisions between meteor-trail classifications are a little arbitrary and somewhat difficult to define: the elusive nature of overdense meteors and difficulties in constraining their masses and sizes as a function of their dependence on initial velocity are best illustrated by considering, for example, a meteor with $M_{\mathrm{v}}=0$. This visual magnitude and its corresponding electron line density will be caused by a body of $20 \mathrm{~mm}$ in diameter and at velocity of $15 \mathrm{~km} \mathrm{~s}^{-1}$, while a body with a smaller diameter of $5 \mathrm{~mm}$ and a larger velocity of $60 \mathrm{~km} \mathrm{~s}^{-1}$ will result in a similar magnitude (Ceplecha et al., 1998). Note that the matter may be further complicated with the inclusion of the effects of the meteoroid density and material properties; however, herein chondritic composition is generally assumed (e.g., Plane, 2012).

The differences in the trail characteristics of the different meteors also extend to temperature and hence rates of diffusion. It is instructive to follow the early development of a meteor trail. The reader may wish to follow Appendix B during this discussion, since it provides a simpler discussion of expected flow dynamics around a meteor head, and includes a relevant diagram. Some of the terms below are better understood through examination of Fig. B1, and discussions of the likelihood of the various features appearing are also discussed there.

In the first stage, meteors ablate as a result of high velocity collisions with atmospheric molecules (Öpik, 1958; McKinley, 1961; McNeil et al., 1998; McNiel et al., 2002; Vondrak et al., 2008). An initial important development is the formation of a heated dense sphere of gas around the meteoroid, referred to as a vapour cloud (e.g. Popova et al., 2000). This in turn leads to subsequent trail (or train) formation and also may lead to shock fronts, as will be considered shortly. The ablated atoms are ionized and the meteor train is considered as a quasi-neutral plasma with approximately the same number of meteoric metal ions and electrons occupying the initial adiabatically formed volume of the meteor trail (McKinley, 1961). The radial distribution of electrons in the trail is assumed to be Gaussian. The density of ionized atoms and electrons in the meteor trail will depend on the ionization coefficient (Kaiser, 1953; Weryk and Brown, 2012, 2013), which varies with element (the coefficient for $\mathrm{Na}$ is much larger than for $\mathrm{Fe}$ or $\mathrm{Mg}$, for example) and velocity (Vondrak et al., 2008). Backscatter meteor radar detects electrons from the specular meteor train (McKinley, 1961).

The typical kinetic energies of ablated meteor atoms are a direct function of the meteoroid velocity, and may exceed several hundred electron volts, while the initial kinetic energy of free electrons may approach $10 \mathrm{eV}$ (Baggaley, 1980). This translational energy, through a series of initially inelastic and then elastic collisions, is converted to heating of the meteor trail and the surrounding atmosphere. The initial collisions in the flow field around and behind the meteor are both elastic and inelastic, depending primarily on the relative kinetic energy and the types of the colliding particles.

As illustrated in Fig. B1, the vapour cloud not only helps in defining the trail but can also lead to shock fronts. The type (and even existence or otherwise) of the meteor related shock fronts can be an important consideration in not only overdense but also strong underdense and transitional meteors. In large overdense meteor trails, both ballistic (in front of the meteor) and bow shocks (generally referred to as cylindrical shocks) will occur, depending on the Knudsen number. Examples are seen in Fig. B1, which gives an overview of the possible structures for a general meteor trail. However, for transitionally dense and underdense trails, while a vapour cloud and ballistic shock front will generally exist, bow shock waves may occur with varying degrees of strength, 
from moderately strong to non-existent. For strongly overdense trails, we can think of the bow shock as a cylindrical discontinuity rapidly expanding radially away from the meteor axis, and conceptually we can visualize the cylindrical shock envelope extending a large distance behind the meteor and also expanding in a direction perpendicular to its axis. For transitionally dense trails the cylindrical shock will be present, but for smaller meteors in this class the front can be quite weak. The weaker underdense meteors have no bow shock at all.

The vapour cloud provides hydrodynamic shielding (with a size generally exceeding the particle size by typically 1 to 2 orders of magnitude (e.g. Boyd, 2000; Popova et al., 2000, 2001; Jenniskens et al. 2004) - Popova et al. (2000) used a value of approximately 20 for the ratio of vapour-cloud diameter to meteoroid diameter), which will be present in all underdense and transitional meteors below $114 \mathrm{~km}$ (Popova et al., 2000, 2001). It is indeed the vapour cloud and the associated hydrodynamic shielding that leads to the formation of an initial ballistic shock front and subsequently the formation of the bow shock front, at least below 95 or $90 \mathrm{~km}$ for transitional meteors, depending on the Knudsen number. These features are discussed further in Popova et al. (2000, 2001) and Campbell-Brown and Koschny (2004).

The mean free molecular path at $80 \mathrm{~km}$ altitude is about $0.6 \mathrm{~cm}$, and that at $90 \mathrm{~km}$ is about $1.5 \mathrm{~cm}$; therefore, since the size of the vapour cloud for a $0.5 \mathrm{~cm}$ meteoroid is typically $10 \mathrm{~cm}$ or more, this amounts to a diameter of 6-16 mean free paths (or more), or a Knudsen number of typically less than 0.1 , which is sufficient to assume an approximately continuous fluid-like flow. In this context, the dense vapour cloud acts somewhat like a blunt solid body, as shown in Fig. B1. (Popova et al., 2000, discuss the fact that a thin layer of the vapour cloud may be drawn off into the surrounding flow, but the bulk of the vapour cloud remains intact - see Appendix B.) Hence, some of the character of the flow around the body can be extrapolated from simulations of fluid flow around high-speed solid bodies, as shown schematically in Fig. B1, although intensities and details of the fronts may vary between situations.

The important points here are that the bow (or cylindrical) shocks may exist but will be considerably weaker in transitional (and strong underdense meteors) than in true overdense meteors, an important point for later in our discussions (see, e.g., Bronshten, 1983; Zel'dovich and Raizer, 2002).

Shocks may have important effects, such as dissociation of the ambient atmospheric molecules which they pass by, but it also needs to be noted that they attenuate rapidly and are only significant within about two initial meteor trail radii of the trail (e.g. Plooster, 1970; ReVelle, 1974).

It is useful to also look in more detail at the flow-field and chemical consequences these fronts. The onset of hydrodynamic shielding (and a subsequent ballistic shock front - for extended discussions, see Bronshten, 1983; Zel'dovich and Raizer, 2002; Anderson, 2006), amplified by meteoric ablation, will greatly affect the consideration of the hypersonic flow in front of and around the hypersonic meteoroid and will be important in our considerations. The formation (or not) of a shock front, for example, will affect the subsequent chemico-physical behaviour of the trail. Since a ballistic shock will ionize and dissociate all atmospheric molecules swept behind it (e.g. Park and Menees, 1978; Menees and Park, 1976), and a bow shock (dependent on its strength) will dissociate many of the molecules (such as ozone) in the near vicinity of the trail (with the impact on air trapped between the trail and the slightly more distant front being of special note), the subsequent chemistry of the trail will be influenced. Additionally, the meteor radar signature may be affected due to possible effects of early electron removal. The strength and persistence of aerodynamic shielding is proportional in size to the cube of the meteoroid velocity (Öpik, 1958; Bronshten, 1983; Jenniskens et al., 2000; Popova et al., 2000; Boyd, 2000; Campbell-Brown and Koschny, 2004) and will alter the flow regime considerations (Boyd, 2000; Popova et al., 2001) and Knudsen number (Josyula and Burt, 2011). As discussed, both overdense and transitionally dense trails may have a ballistic shockwave front, with the temperature of the shock region being as high as $10000 \mathrm{~K}$ or higher (e.g. Boyd, 2000). The shock forms a paraboloid about the head and flows back as a conical (near cylindrical) section, with the angle of the cone from the trajectory being of the order of $\tan ^{-1}\left(c_{\mathrm{s}} / v_{\mathrm{m}}\right), c_{\mathrm{s}}$ being the speed of sound and $v_{\mathrm{m}}$ being the meteor speed (this can be shown by simple geometric considerations). For a meteor travelling at $40 \mathrm{~km} \mathrm{~s}^{-1}$, this angle is $\sim 0.5^{\circ}$. In other words, the angle of the front relative to the trail is quite small, usually well under $1^{\circ}$.

The cylindrical shock wave in typical overdense and some transitional meteors is sufficiently strong that it results in a near-instantaneous rise in temperature immediately behind the shock front, with the temperature rise being of the order of $6000 \mathrm{~K}$. The subsequent exchange of translational, rotational, and vibrational energy between atmospheric and ablating meteor constituents trapped within the flow-field behind the meteor will bring the temperature in the region further back along the meteor wake down to about $4400 \mathrm{~K}$ (Jenniskens et al., 2004). In addition the cylindrical shock front surrounding the trail (and the associated high temperatures) can dissociate many molecules in the ambient air situated between the trail and the front as it radially propagates away from the meteor axis, and also as the meteor (and wake) move forward into the air ahead of the meteor. The trail may then diffuse into this region at a later time. The scavenging achieved by the shock front can thus impact the likelihood of various chemical reactions. Jenniskens et al. (2004), Jenniskens and Stenbaek-Nielsen (2004), and Jenniskens et al. (2000) have discussed evidence for these fronts and measured temperatures associated with the meteor (although no lateral resolution was available). The processes within the wake and immediate train of the meteor trail have been dis- 
cussed further by Menees and Park (1976), Park and Menees (1978), and Berezhnoy and Borovička (2010).

Another point to note, which will be important, is the rate of cooling of the trail. This will affect the ambipolar diffusion rate, which will itself be time-varying. This point will be further considered in the next section.

Returning to the first stage of the overdense and transitional meteor trail evolution, we note that it encompasses the initial radiative energy loss phase of the meteor plasma, where a substantial translational velocity difference of more than $5 \mathrm{~km} \mathrm{~s}^{-1}$ exists between the plasma in the radiating volume and the ambient air in the direction of the meteoroid's axis (Jenniskens et al., 2004). In general, the ablated meteor vapour and plasma, initially moving at meteor velocities, will stop in a few hundred metres, where the stopping distance is a function of the meteoroid initial velocity. This stage of ablated meteor vapour and plasma dynamics is accompanied by adiabatic (explosive) meteor trail formation with some initial radius $r_{0}$ (Jones, 1995; Jones and Campbell-Brown, 2005); the process is discussed in Appendix B in the context of a "neck" of recompressed air behind the meteoroid. The explosive adiabatic formation of the initial trail with $r_{0}$ is accompanied by turbulence generated in the immediate meteor wake $(T<10000 \mathrm{~K})$, driven by the local flow field velocity, temperature, and density gradients (Lees and Hromas, 1962). This process is completed within less than the first millisecond. Values of $r_{0}$ will be discussed subsequently. The initial radius, and the nature of the fronts produced, will be topics of significance later in this paper. It should be noted that some computer models of meteor trail formation do not form a neck (e.g. Boyd, 2000), and it is unclear whether this is a function of the size of the meteoroid or the nature of the model. Small meteors might not be expected to form a neck. However, the vapour cloud, and some form of ballistic shock, should be relatively ubiquitous.

\section{Classes of trails observed}

The purpose of this section is to determine the range of nonunderdense meteors covered by the radars. This is largely discussed in Appendix C, but here we give a brief summary.

For an assumed cylindrical trail with Gaussian cross section, the line density relates to the peak volume density through the relation

$N_{\ell}=\pi a^{2} N_{0}$,

where $a$ is the radius at which the volume density falls to $1 / e$ of the peak volume density $N_{0}$. The plasma frequency of the trail at its peak electron density at time $t=0$ is given by

$f_{\mathrm{p}}=e /\left(2 \pi r_{0}\right) \sqrt{ }\left(N_{\ell} / \pi \varepsilon_{0} m_{\mathrm{e}}\right)$,

where $e$ is the electronic charge, $r_{0}$ is the initial radius, $\varepsilon_{0}$ is the permittivity of free space and $m_{e}$ is the electron mass.
If the radar frequency is less than $f_{\mathrm{p}}$, then the trail will be critically reflecting and will appear overdense.

Using values of $r_{0}$ from Baggaley and Fisher (1980), the plasma frequencies are shown in column 4 of Table $\mathrm{C} 1$ for different altitudes and line densities.

In Appendix $\mathrm{C}$ we then consider the expansion of the trail and calculate the 1/e radius $a$ at which the peak plasma frequency drops below $35 \mathrm{MHz}$. At this point the trail becomes underdense for a $35 \mathrm{MHz}$ radar. If the trail is already underdense at $t=0$, then the trail decays exponentially and reaches half of its initial amplitude when $a=0.195 \lambda$. The trail can be considered to have all but disappeared once $a$ reaches $0.4 \lambda$. However, for cases where the initial peak value of $f_{\mathrm{p}}$ exceeds the radio frequency, the radar signal is reflected from the edge of the trail, at the point where $f_{\mathrm{p}}$ equals the radar frequency. This will happen regardless of the value of $a-$ even if $a$ is many times $\lambda$. The strength of the backscatter signal will be, to first order, invariant during this process, except for wobbles associated with distortions of the trail, perhaps due to local wind shears and background turbulence. However, as soon as the peak plasma frequency drops below the radar frequency, the trail becomes fully transparent and will instantly disappear from the view of the radar as long as $a \gtrsim 0.4 \lambda$. Hence, the lifetime of any initially non-underdense trail will be close to the time taken for the peak plasma frequency to drop below the radar frequency.

Typical values for the trail duration based on the above arguments are shown in column 8 of Table $\mathrm{C} 1$, assuming that diffusion takes place at the ambient rate. The classical formula for diffusion times is from Greenhow (1952) and is

$\tau=r_{\mathrm{e}} /\left(4 \pi^{2}\right) N_{\ell} \lambda^{2} / D_{\mathrm{a}}$,

where $r_{\mathrm{e}}$ is the electron radius $\left(2.8 \times 10^{-15} \mathrm{~m}\right)$, and $N_{\ell}$ is the line density. For $N_{\ell}=10^{16} \mathrm{~m}^{-1}$ and $\lambda=8 \mathrm{~m}$, this is

$\tau=45.4 / D_{\mathrm{a}}$.

Agreement between our calculations and this expression is good to about $10 \%$ or better (Greenhow considered $r_{0} \sim 0$ ). Lifetimes vary between fractions of a second and tens of seconds.

But a problem arises here. While it is common to take $D_{\text {a }}$ as the ambient ambipolar diffusion rate, this is a serious error. For non-underdense meteors, the situation involves a hot trail of ions diffusing through a colder background plasma, dragging the electrons with the ions to maintain general charge neutrality. As shown in Appendix C, the proper diffusion coefficient to use is the geometric mean of the diffusion rates of the plasma and the ambient atmosphere. Column 9 gives more appropriate diffusion coefficients, based on assumed temperatures as discussed in the Appendix. Lifetimes are now of the order of tenths of a second to several seconds (column 10).

Lifetimes of more than $2 \mathrm{~s}$ will not be identified as meteors by the SKiYMET radar due to its selection criteria. Hence, 
Table 1. List of radar sites, geographical coordinates, operating frequencies, and years of available data.

\begin{tabular}{lrrr}
\hline Site & Coordinates & $\begin{array}{c}\text { Operating } \\
\text { frequency } \\
(\mathrm{MHz})\end{array}$ & $\begin{array}{c}\text { Years } \\
\text { covered } \\
\text { (incl.) }\end{array}$ \\
\hline Resolute Bay, Nunavut, Canada & $74.7^{\circ} \mathrm{N}, 94.9^{\circ} \mathrm{W}$ & 51.50 & $1997-2015$ \\
Yellowknife, NWT, Canada & $62.5^{\circ} \mathrm{N}, 114.5^{\circ} \mathrm{W}$ & 35.65 & $2002-2015$ \\
Socorro, New Mexico, USA & $34.1^{\circ} \mathrm{N}, 106.9^{\circ} \mathrm{W}$ & 35.24 & $2002-2015$ \\
Santa Cruz, Costa Rica & $10.3^{\circ} \mathrm{N}, 85.6^{\circ} \mathrm{W}$ & 35.65 & $2005-2013$ \\
\hline
\end{tabular}

meteor trails with line densities greater than the overdense cut-off of $10^{16} \mathrm{~m}^{-1}$ will only be seen at altitudes above about 90-95 km (Table C1, column 10), and normally the vast majority of meteors seen in our studies will have line densities less than about $3 \times 10^{15} \mathrm{~m}^{-1}$.

Hence, we have a very important result - namely that most of the non-underdense meteors measured in these experiments will be transitionally overdense and not fully overdense. The significance of this will become clear later in our discussions about mechanisms. We will also show that our measured decay times broadly match the range of values shown in Table $\mathrm{C} 1$, especially for the case of critically transitional meteor trails (i.e. cases where $N_{\ell}=2.4 \times 10^{14} \mathrm{~m}^{-1}$ ).

\section{Radar designs and locations}

Four radars were used in this study, at sites ranging from near-equatorial to the Arctic. The sites are listed in Table 1, where the radar frequencies and site locations are given, along with the years of data covered. Three of the radars had frequencies close to $35 \mathrm{MHz}$, while the Resolute Bay system operated at $51.5 \mathrm{MHz}$. All sites used software written for the SKiYMET radars, as described in Hocking et al. (2001). Protocols used for meteor detection are outlined there. Of primary importance are the following. First, a likely meteor is initially stored as a $4 \mathrm{~s}$ sequence of data, beginning $1 \mathrm{~s}$ before the suspected meteor is first detected. Further analysis is then applied, in which the signals on five spaced antenna receivers are cross-correlated and inspected for consistent phase frequency behaviour. No requirement is made that the amplitude should follow an exponential decay: rather, the software looks for a rapid turn-on followed by a general decrease over typically the next $2 \mathrm{~s}$. Small bumps and dips are allowed, provided they are not too excessive (as specified in Hocking et al., 2001). This means that underdense meteors (with typical exponential decays), transitionally dense and even some overdense meteors (with a more boxcar-like, slightly decaying, behaviour) can in principle be identified by the system.

When the system is left running with the transmitter turned off, very few meteor detections are generally made over periods of typically a day, so the system has good discrimination against lightning and other natural impulsive events. The system can, however, be sensitive to interference from other transmitters of appropriate frequency. At the Socorro site, typically four false detections of radio transmitters occur in every 10000 unambiguous meteor detections. At Costa Rica, where frequency allocation by government authorities is less well implemented, about four false meteors are detected for every 1000 true meteor detections. These figures will become relevant later in the text.

The limit of $2 \mathrm{~s}$ to the detectable lifetime also deserves a brief comment. Generally, our measured maximum recorded lifetimes are less than $1.5 \mathrm{~s}$, i.e. less than this $2 \mathrm{~s}$ limit. However, as a further test, the software at the Resolute Bay site was modified to allow meteors of unlimited duration to be detected (at least up to about $10 \mathrm{~s}$ ). The requirements for rapid turn-on, good amplitude, and phase correlation between receivers, as well as generally decreasing amplitude over time, were retained. During these tests, typically one to two additional, longer-lived meteors were detected during the course of a day (during which typically 2000 shorter-lived meteors were detected), indicating that the upper limits shown in the data below are not artefacts of the analysis but are true upper limits dictated by the properties of the trails in backscatter situations.

Other details about the radar design can be found in Hocking et al. (2001). For the purposes of this paper, it is only necessary to know that all meteors used in this study could be unambiguously located in angle and in height: angular accuracy varied from typically 0.5 to $1.5^{\circ}$ (depending largely on zenith angle), and range accuracy was of the order of $2 \mathrm{~km}$.

\section{Data analysis methods}

\subsection{Primary tools}

As our primary tool of analysis, we will use the complementary cumulative distribution of decay times $\tau$, in which we plot the number of meteors with a half-amplitude decay time greater than a value $\tau$ vs. the value of $\tau$ itself. An example is shown in Fig. 1. In this case, we illustrate examples for the site of Yellowknife, using accumulated data over 14 years (from 2002 to 2015) for selected summer and winter months, and for the height intervals 80 to 83 and 89 to $92 \mathrm{~km}$. The 80 to $83 \mathrm{~km}$ data include meteors at $80.01 \mathrm{~km}$ height but not $80.00 \mathrm{~km}$, up to and including $83.00 \mathrm{~km}$. Likewise, the 89 $92 \mathrm{~km}$ data include $92.00 \mathrm{~km}$ data but not $89.00 \mathrm{~km}$. Plots are normalized to 100000 detections, as explained in the caption. 

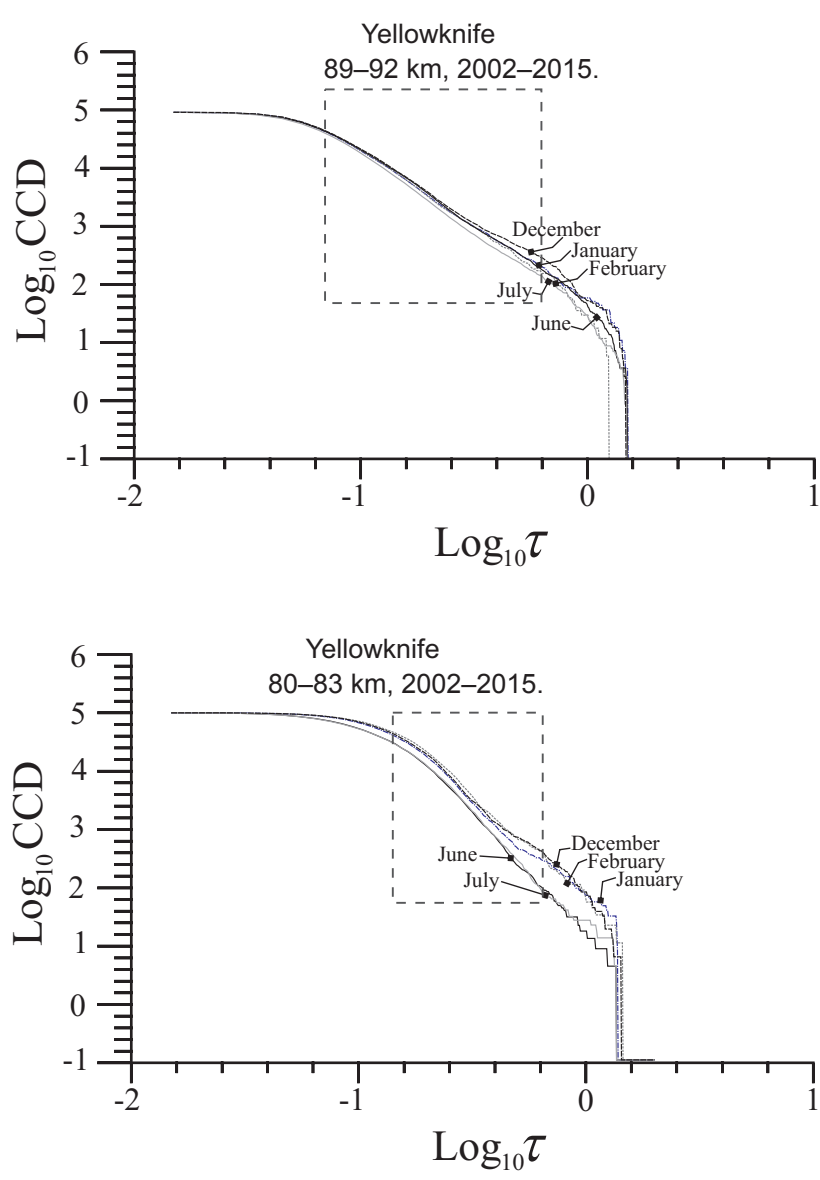

Figure 1. Complementary cumulative distributions of decay times for the Yellowknife site over a time period from 2002 to 2015 for summer (represented by June and July) and winter (represented by December to February), for heights of 89 to $92 \mathrm{~km}$ and 80 to $83 \mathrm{~km}$. Values are normalized in such a way that the total number of detections is treated as 100000 meteors, which is representative of the number of meteors detected in a $3 \mathrm{~km}$ deep bin at 89 to $92 \mathrm{~km}$ for the month of June over the full time interval. At other heights and in other months the number can be lower by 5 to 10 times, but the normalization allows easy comparison between datasets. The knees in the plots are taken to have a line density of $2.4 \times 10^{14}{\text { electrons } \mathrm{m}^{-1}}^{-1}$ (see Appendix C), and meteors to the right of the boxed areas shown

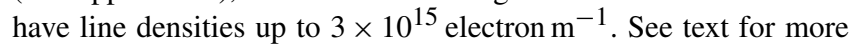
details.

Individual histograms were also prepared and had broadly Gaussian distributions with modal values in the range 0.03 to $0.07 \mathrm{~s}$ at $90 \mathrm{~km}$, and 0.06 to $0.12 \mathrm{~s}$ at typically $82 \mathrm{~km}$ altitude. Summer and winter data have been chosen for reasons to be discussed shortly - data for all months of the year exist and will be discussed in due course.

The data chosen in Fig. 1 have been selected for several reasons. First, the choice of months allows us to examine extremes due to temperature and pressure effects. Yellowknife is at a near-Arctic latitude, so it experiences extremes of temperature from about $160 \mathrm{~K}$ in summer to about $240 \mathrm{~K}$ in winter at $85-90 \mathrm{~km}$ altitude (e.g. Garcia and Solomon, 1983; Plane et al., 2015). In addition, summer and winter are times of low ozone density in the $80-90 \mathrm{~km}$ region (see, e.g., Thomas, 1990: also demonstrated later in this text; Fig. 5a). Choosing these months allows us to somewhat reduce the impact of ozone chemistry on our plot, although of course the impact of other reactive compounds like NO and hydrated water clusters, dust, etc. cannot all be excluded.

The decay times for underdense meteors are proportional to $P / T^{2}$, where $P$ is pressure and $T$ is temperature. The relation between the two parameters $P$ and $T$ is not trivial, as shown in Hocking (1999a), and indeed different empirical models give different seasonal variations. Nevertheless, the seasons of summer and winter represent extremes, so variations of the complementary cumulative distribution (CCD) between these months can be considered to be most affected by pressure and temperature variations. Yet examination of the upper curves in Fig. 1 (Yellowknife, 89-92 km) shows few distinguishable differences between the summer and winter data within the boxed area. The lower curves (Yellowknife, $80-83 \mathrm{~km}$ altitude) show quite noticeable differences between the summer and winter data within the boxed region, with the winter data shifted to the right. However, significantly, the summer and winter datasets show the same slopes in the left-hand half of the boxed region, a point that will become important later.

To the right of the boxed area, in the extreme tail, there are noticeable differences between the months at $80-83 \mathrm{~km}$ altitude, with the wintertime counts being much higher. This is noteworthy, but the statistics are poor here - typically there are fewer than 100 normalized accumulated meteors at decay times in excess of $1 \mathrm{~s}$, so while they are of interest, we feel that these data are too few to use reliably. These observations of the character of the summer vs. winter behaviour in the boxed area, and in the tail of the CCD plots, will be of value later in the text.

Now we turn to discussion of Fig. 2 (Socorro, midlatitude). In this case, complementary cumulative distributions have been plotted for each month of the year, accumulated over all the years from 2002 to 2015 . The data have been divided into night and day. It is clear that, to the right of $\log _{10} \tau=-0.5$, the night-time counts are substantially suppressed, with the suppression increasing as $\tau$ increases. This is particularly evident at the lower heights, as discussed in the caption.

Another noteworthy point that can be seen in Figs. 1 and 2 is a distinct knee in the CCD where the curve bends from relatively flat on the left to more steeply descending on the right. Approximate values of the decay time associated with the bend in the CCD's for Socorro are given as bracketed quantities in the last column of Table $\mathrm{C} 1$. It can be seen that these values match the decay times expected for the transition between underdense and transitionally dense meteor trails to within a factor of better than 2, leading us to associate this knee of the CCD with this transitive temporal scale. Never- 

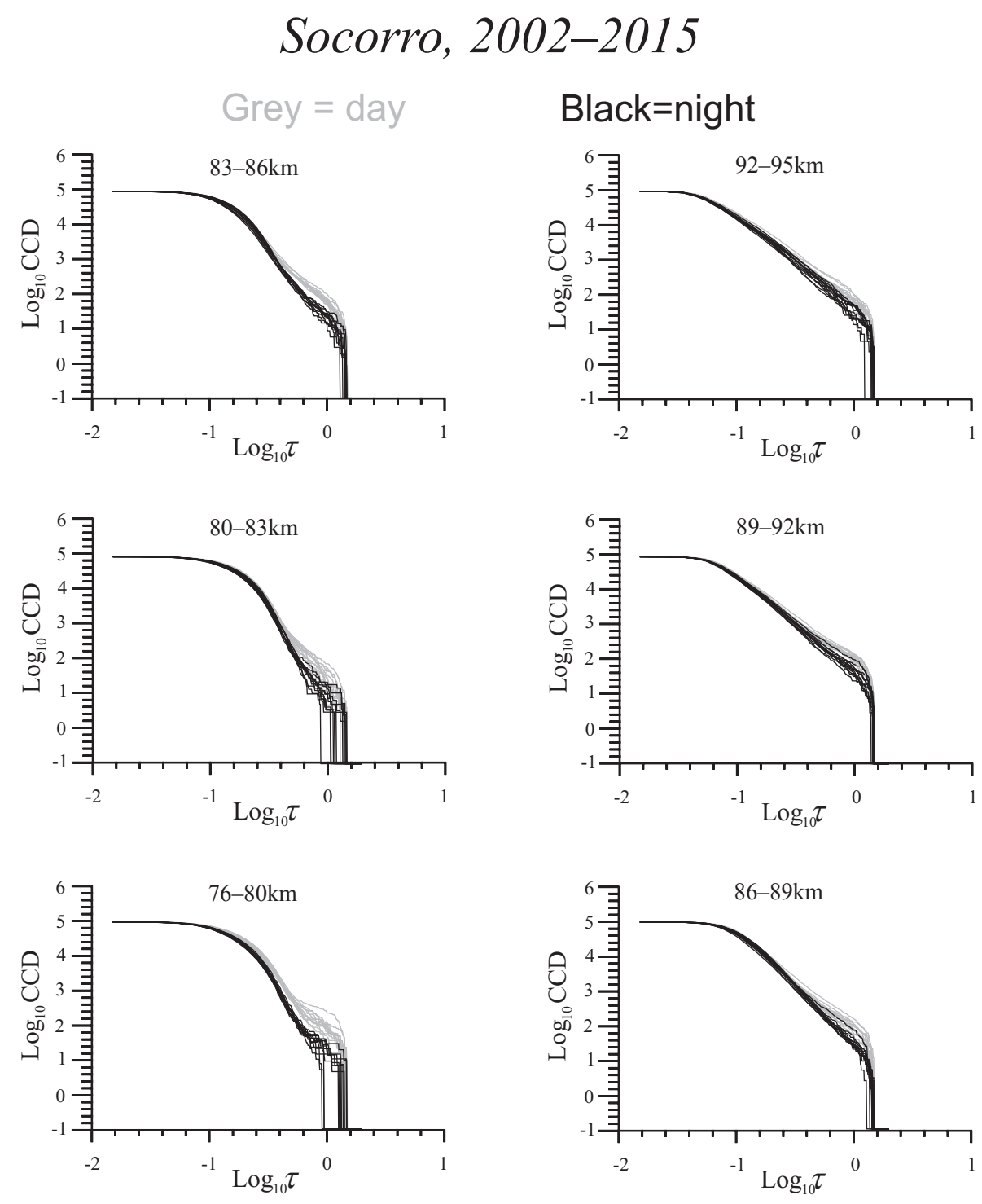

Figure 2. Complementary cumulative distributions for Socorro, New Mexico, USA, for 2002 to 2015. Each line corresponds to 1 month, with data accumulated for that month over all years. Data have been separated into day (grey) and night (black). Data are normalized as in Fig. 1. There are therefore 12 separate grey lines (one for each month) and 12 separate black lines (also one for each month) in each height bin. It is clear that, in general, the normalized counts for night-time are suppressed relative to daytime to the right of $\log _{10} \tau=-0.4$ for all heights, with the suppression being greatest at the lower heights and the largest decay times.

theless, while the association is interesting, it is not crucial to our subsequent discussions.

In contrast to Figs. 1 and 2, Fig. 3 shows the case for a lower set of heights $(80-83 \mathrm{~km})$ at Costa Rica. Rather than a distinct day-night difference, the day and night CCDs are very similar, apart from the two special months of February and September. This case is unusual compared to the other sites but is nonetheless important and will be discussed further in due course.

\subsection{Chemical decay times}

The differences seen between day and night in the previous figures are not indicative of dominance by any one process. Appendix $\mathrm{C}$ has shown that the lifetimes observed for the non-underdense meteor trails are not very different to values expected from ambipolar diffusion. Therefore, the process that accounts for the day-night differences at low and mid-latitudes - and, by extension, the seasonal variations at subarctic and Arctic latitudes - is modest.

We therefore consider that there are two processes involved: diffusion, defined largely by the daytime lifetimes, 


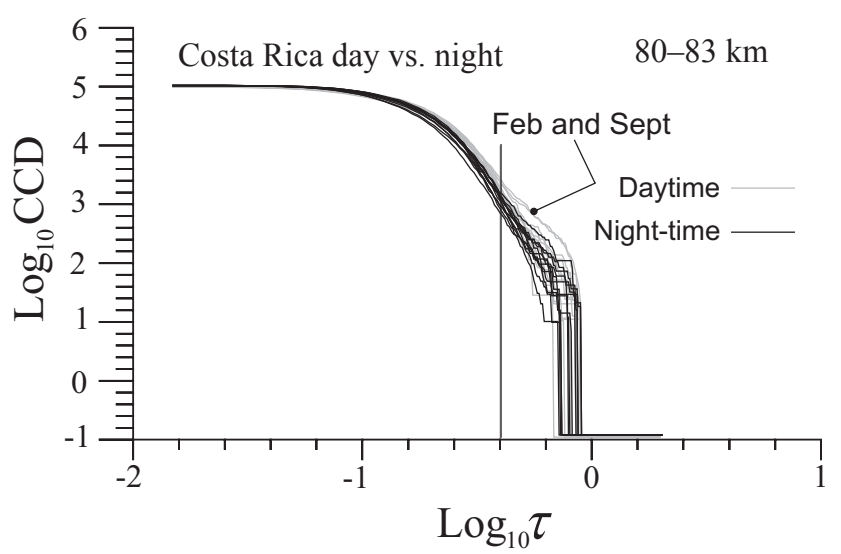

Figure 3. CCDs for Costa Rica at $80-83 \mathrm{~km}$ altitude for all months over all years of observations. Data are grouped according to daytime (grey) and night-time (black). Twelve graphs of each shade (grey or black) appear, one for each month of the year, but the months are not otherwise distinguished. Two unusual months are shown (February and September). In this case, the day and night CCDs are harder to separate, but differences do exist, as will be seen later in Fig. 8. See text for further discussion.

and chemical depletion which makes the night-time trails disappear slightly more rapidly than the daytime ones. We assume that the decay rate of each process is approximately exponential, which is a common feature of both diffusion and first-order chemical reaction kinetics, and assume that the two exponential decays are multiplicative. Correspondingly the first-order decay rates, expressed as the reciprocal of the time constants, are additive. We recognize this is an approximation, and may not always be valid, but it is sufficient for the following calculations. We may then write that

$\exp \left\{-t / \tau_{\text {night }}\right\}=\exp \left\{-t / \tau_{\text {day }}\right\} \times \exp \left\{-t \tau_{\text {chem }}\right\}$

so that

$1 / \tau_{\text {chem }}=1 / \tau_{\text {night }}-1 / \tau_{\text {day }}$.

The same formula applies if half-amplitude decay times are used throughout. We have taken the CCDs and found selected values of the daytime decay time at values of $0.255,0.305$, $0.355, \ldots, 0.555$, and 0.605 , and then found the matching night-time decay times with the same value of the normalized CCD. We have then applied the above equation to determine $\tau_{\text {chem }}$ in all months and in all height bins. The results are shown in Table 2. No real trend as a function of height is found, but there is general consistency in the numbers, with a mean decay time of $4.0 \mathrm{~s}$. Reasons for this decay time will be discussed later, but we hasten to add that this is not in reality a simple process: we will see that we need to concern ourselves about the available reservoir of quenching molecules, and even the impact of shock fronts and background atmospheric diffusion. The general temporal variation of the meteors is also not always the same. Hence, the
Table 2. Mean "chemical" decay times for different height regimes for Socorro, determined by using day-night differences from Socorro. Errors represent 1 standard error. See text for details.

\begin{tabular}{lrr}
\hline $\begin{array}{l}\text { Height } \\
\text { range } \\
(\mathrm{km})\end{array}$ & $\begin{array}{r}\text { Mean (all } \\
\text { months and } \\
\text { decay times })\end{array}$ & $\begin{array}{r}\text { Standard } \\
\text { error }\end{array}$ \\
\hline $76-80$ & 2.7 & 0.1 \\
$80-83$ & 4.5 & 0.3 \\
$83-86$ & 4.0 & 0.3 \\
$86-89$ & 4.3 & 0.3 \\
$89-92$ & 3.9 & 0.4 \\
$92-95$ & 4.3 & 0.5 \\
\hline
\end{tabular}

value of $\tau_{\text {chem }}$ should be considered as an estimate rather than a precise value. For now, this simply gives an idea of the degree of importance the additional chemistry, which is clearly modest but measurable.

\subsection{Another parameter for display purposes}

Before examining the likely reasons for this chemical component of the decay, we need to consider some suitable parameterizations of the day-night differences. For Socorro and Costa Rica, meaningful parameterization of the impact of the chemistry can be obtained by comparing daylight and night-time values. We have already considered the differences in daytime and night-time values of the decay time at fixed CCD values for various daytime decay times (Sect. 5.2).

An alternative approach might be to choose particular values of $\log _{10} \tau$ and study differences in the CCD values at that point. For example, $\log _{10} \tau=-0.3$ or -0.2 or even $0.0 \mathrm{might}$ be used. This was also done in the analysis of the data - differences were recorded at $\log _{10} \tau=-0.3,-0.2$, and -0.1 ( $\tau$ values of $0.5,0.6$, and 0.8 respectively). Another possibility might be to integrate the differences between the two curves from the far left to, say, $\log _{10} \tau=-0.4$, but in fact the value of the CCD at -0.4 is already an integral of all the points in the histogram to the right of it, making for a double integral, which seems excessive.

However, these ideas have limits. First, they deal with very low number of counts - as low as 100 or so - and thus may contain statistical unreliability. But, more importantly, such procedures limit the ability to process Arctic data, since at some times of the year there is no night, and at other times of year there is no day. At Yellowknife and Resolute Bay, periods of equally distributed daylight and darkness occur only around the equinoxes, and even then the process is complicated by long hours of twilight. Thus, we sought out a parameter which might be independent of applying day-night differences.

It was decided to determine the slopes of the monthly curves evaluated around the point $\log _{10} \tau=-0.4$. Specifi- 


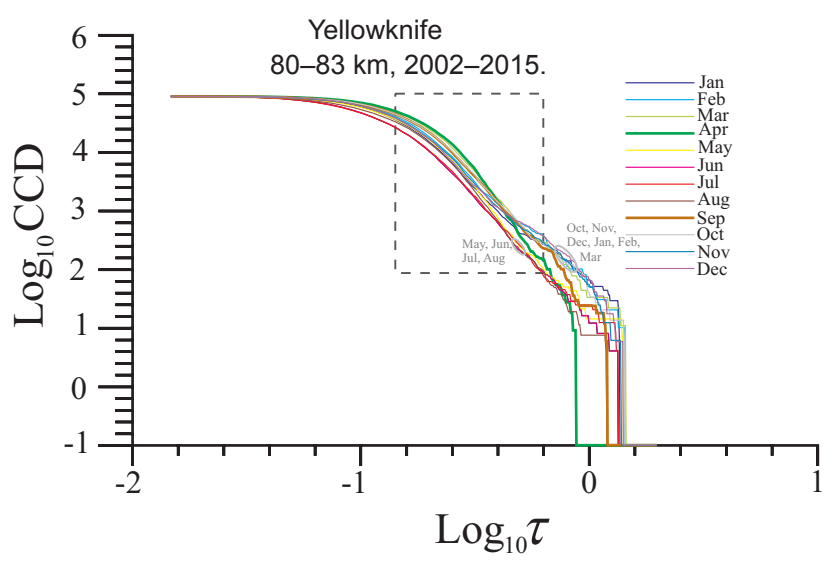

Figure 4. This figure shows a repeat of Fig. 1, but this time showing all months of the years. Notice that the months May to August form one cluster (summertime) and October to March form another (wintertime), but the months of April and September show a distinctly different behaviour. April in particular stands out and is highlighted with an extra-thick green line. In particular, the slope of this green line in the range $-0.6<\log _{10} \tau<-0.2$ is clearly much steeper (more negative) than the slopes for the other months. The same is true, but to a lesser extent, for the month of September.

cally, it was evaluated between the points -0.5 and -0.3 for Costa Rica, Socorro, and Yellowknife. For Resolute Bay, the band from -0.6 to -0.4 was used, due to the radar's different radio frequency. A visual examination of Fig. 2 shows that, at this point, the night-time slopes are considerably steeper (more negative) than the daytime slopes.

To further emphasize the potential of using these slopes, we also present Fig. 4. This is a repeat of Fig. 1, but this time all months of the year are displayed. May to August form one cluster (summertime), similar in general form to the months of June and July shown in Fig. 1, while the data covering October to March complete another cluster (wintertime) similar to December-January-February in Fig. 1. However, the months of April and September show a distinctly different behaviour and, in particular, have more negative mean slopes in the range from $\log _{10} \tau=-0.6$ to -0.2 .

Based on the above discussion, we have adopted the bestfit slope in the region $-0.5<\log _{10} \tau<-0.3$ as our diagnostic of the behaviour of the tail of the CCD at the southern sites, and -0.6 to -0.4 for Resolute Bay. Use of such a parameter does not require separate daylight and night-time hours in any one day.

The various characteristics discussed above (especially the day-night differences at mid-latitudes and seasonal variations in the Arctic) suggest the possible involvement of ozone in the non-underdense meteor early-stage depletion process. Day-night variations at Arctic latitudes may also be of interest, but only for brief periods of time at the equinoxes; we will not discuss these here but will return to this matter later. At this point we will demonstrate this relation with the lim-
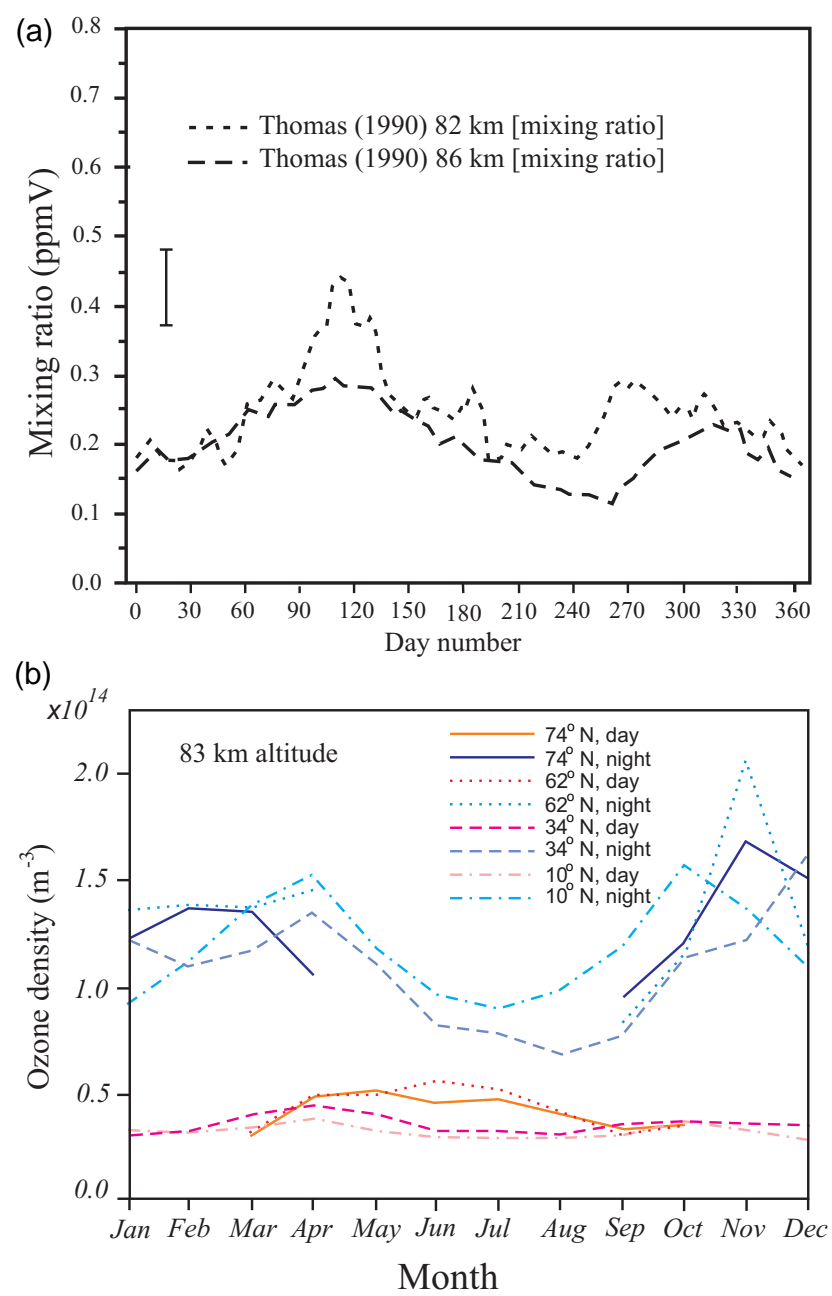

Figure 5. (a) Plots of satellite-measured ozone volume mixing ratio (parts per million by volume) at 82 and $86 \mathrm{~km}$, at $45^{\circ} \mathrm{N}$. Increases in ozone ppmv in spring and autumn are evident. (b) Night and daytime seasonal variations for $\mathrm{O}_{3}$ density from the WACCM model at $83 \mathrm{~km}$ altitude, for latitudes close to the four radar sites. The upper set of graphs is for night-time, and the lower set is for daytime. Values were obtained at local midnight ("night") and midday ("day"). Our interest here is in solar effects, so for the two more northerly latitudes (Yellowknife and Resolute Bay) daytime data have been removed for November through to February (since the sun is largely absent in those months), while night-time values have been removed for May through August (since darkness is relatively rare in those months at high latitudes). Similar trends to (a) may be seen, with lowest overall values in summer. Both (a) and (b) show peaks in night-time activity around the equinoxes, though (a) shows a more pronounced local minimum in winter months.

ited dataset presented to date, and then will go on to further verify the results with a much larger dataset.

To begin, we consider seasonal and day-night differences. It is expected from a variety of studies - both theoretical and experimental - that ozone densities in the mesosphere will be highest at night and lowest during the day, and that seasonal 
variations should tend to show maxima at the equinoxes. Typical experimental seasonal values are seen in Fig. 5a. Modelled ozone is shown in Fig. 5b, where results have been separated into daytime and night-time values. For periods when the Arctic day was all darkness (winter) or all sunlit (summer), only the appropriate day/night data are shown.

The modelled data were determined using the Whole Atmosphere Community Climate Model (WACCM4; Marsh et al., 2013) where the meteorological reanalyses from the European Centre for Medium-Range Weather Forecasts (ECMWF) Re-Analysis (ERA)-Interim (Dee et al., 2011) were used to specify the dynamics below $50 \mathrm{~km}$. The model simulation was run from 2003 at a horizontal resolution of $1.9^{\circ} \times 2.5^{\circ}$ (in latitude and longitude respectively) and a hybrid $\sigma$-pressure vertical coordinate with 88 levels (1000$5.96 \times 10^{-6} \mathrm{hPa}$ ) was used which covered an altitude range from the surface to $\sim 145 \mathrm{~km}$. The vertical resolution in the mesosphere and lower thermosphere (MLT) was about $3.5 \mathrm{~km}$. The global fields from the model simulation were saved every $12 \mathrm{~h}$ and the model output was interpolated to fixed altitudes above $50 \mathrm{~km}$ at $1 \mathrm{~km}$ vertical intervals for analysis. As a point of interest, WACCM data do appear to underestimate $\mathrm{O}_{3}$ by a mean factor of 2 compared to SABER observations (e.g. Smith et al., 2015). However, the relative $\mathrm{O}_{3}$ changes predicted by WACCM should be generally sufficient for our discussions here.

The day-night differences are evident in Fig. 5b. Additional confirmation can be found in Fig. 7 of Smith and Marsh (2005) and Figs. 3 and 4 of Rogers et al. (2009). Day-night variations are of the order of a factor of 4 in Fig. 5b, with night-time values being highest. These results are broadly consistent with our Fig. 2. The day-night differences at upper heights can be as high as a factor of 10 (Smith and Marsh, 2005), again with larger values occurring at night.

To date we have not discussed height variations, though all studies (including WACCM) show a maximum in ozone densities at $\sim 86$ to $\sim 90 \mathrm{~km}$ altitude (depending on latitude, with the peak being generally highest at the Equator). We have concentrated on the lower altitudes, since our trail depletions seem graphically most prominent below $86 \mathrm{~km}$ altitude. However, it needs to be kept in mind that, if ozone chemistry is indeed involved, the relation between trail depletion rates and ozone density may not be one to one. The efficiency of ionization of the trail is important, and of course reaction rates depend on species concentrations, possibly including mean atmospheric densities (depending on the process). For now we will not concern ourselves too much with the heights, and we will concentrate mostly on temporal variations. Height variations will be discussed in more detail in the next section.

Importantly, the night-time maximum in ozone matches the more rapid depletion of the non-underdense meteor trails at night-time shown in Fig. 2.

Another point of note is the seasonal behaviour. Fig. 5a shows the average latitudinal annual variation of ozone vol- ume mixing ratios in ppmv (from Thomas, 1990), and peaks in April and September/October are evident, while Fig. 5b shows ozone density results from the WACCM model. Measurements of ozone for the mesosphere from satellites require significant averaging in order to reduce uncertainties, and of course models are restricted to some extent by the nature of the input data (often satellite-based) and the accuracy of the physics and chemistry embedded in the model. Thus, both datasets may have deviations from reality. Regardless, the fact remains that a clear maximum in ozone density is apparent in April and to a lesser extent in September/November. This matches the special cases of significant depletion in meteor trail lifetimes in April and September in Fig. 4.

With these tentative inverse correlations in nonunderdense meteor trail lifetimes and ozone density, we are in a position to see whether the relation is more general.

\section{Expanded studies}

In this section we will look at the behaviour of the slopes deduced using the procedure discussed above as a function of time of year, as well as day-night differences. The two main graphs for discussion are Figs. 6 and 7. In Fig. 6 (the two Arctic sites), contours of the slopes are shown on the left. For these Arctic sites we have used all-day ( $24 \mathrm{~h})$ datasets, and the reader should recognize that in summer the sun is almost always up, while in winter there is largely $24 \mathrm{~h}$ of darkness at these sites. The most dramatic ozone variations at Arctic latitudes are seasonal, with strong maxima at the equinoxes, as discussed with respect to Fig. 5.

Figure 7 shows data for the mid-latitude and equatorial sites (Socorro and Costa Rica). Here data are separated into daytime (sunlit) and night-time (darkness). Correct sunrise and sunset times were used to distinguish the data. The upper four graphs in Fig. 7a, b, d, and e show slopes for daytime and night-time, as indicated. The slopes are clearly more negative (steeper) at the lower heights at all times but are even steeper at night.

Returning to Fig. 6, the steepest slopes clearly occur in March to May and September/October above both Yellowknife and Resolute Bay. Interestingly, a height progression with increasing height is apparent, with the minima at $82-83 \mathrm{~km}$ altitude being deepest in the later months of the northern spring, and in late summer as well as the earlier months of autumn, while minima at lower heights occur earlier in spring and later in autumn.

The right-hand graphs of Fig. 6 show the modes and mean decay times for all meteors (including underdense). This is done as a check to ensure that the impact seen in the tail is not an artefact of the main part of the CCD. It is clear that the mean and modal decay times actually show a maximum at around the same time as the minima in slopes appear and that they even have a similar temporal and altitudinal variation in spring and autumn as shown in Fig. 6a and b. How- 

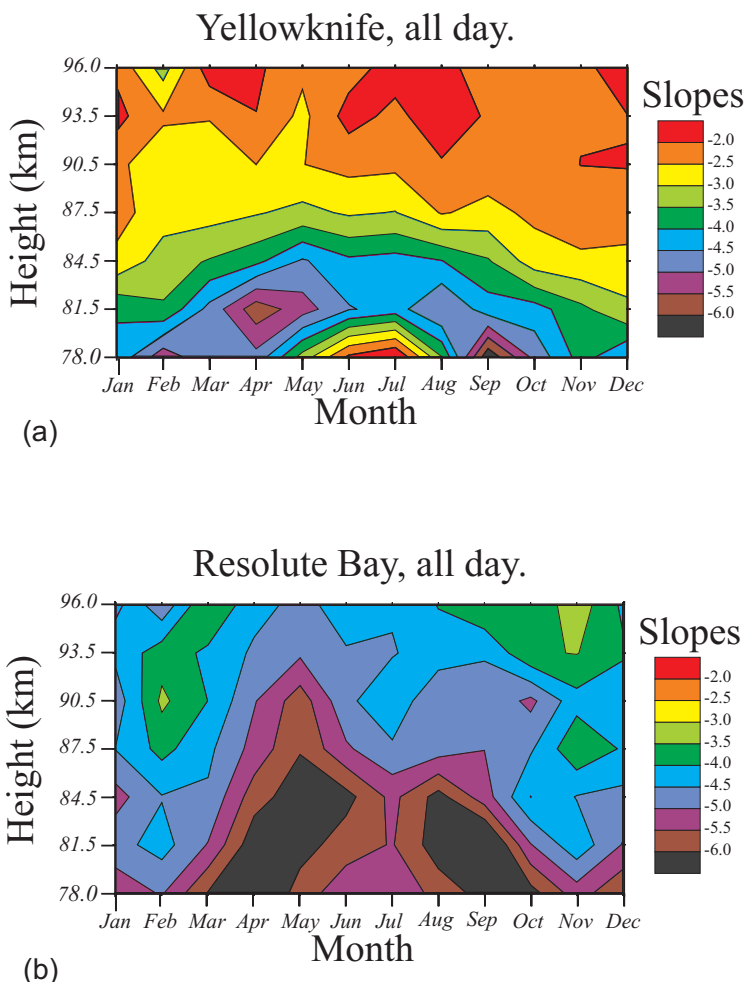

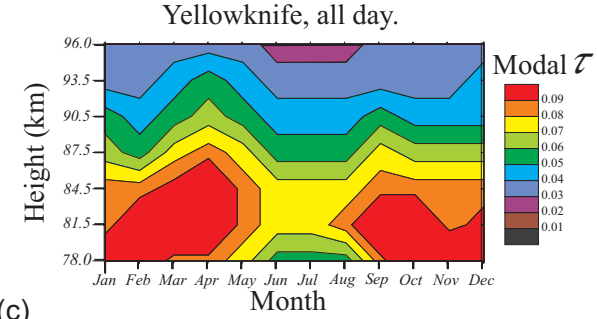

(c)

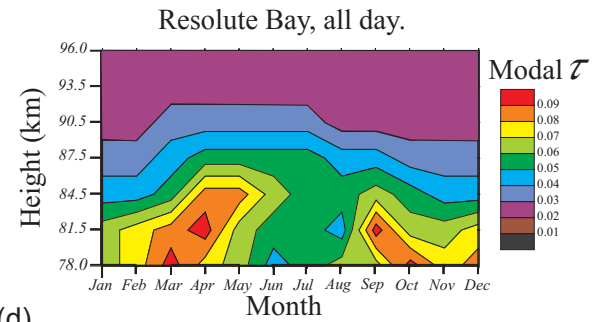

(d)

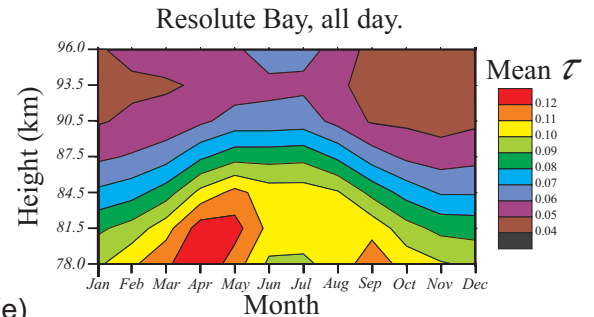

Figure 6. Panels (a) and (b) show contour plots of the slope of the tail of the CCD, as described in the text, as a function of height and month, for Yellowknife and Resolute Bay respectively. Data cover the full range of available years. Panels (c) to (e) show contour plots of the modes and means of the total distribution, as described in each figure. These last three plots do not pertain directly to the non-underdense meteors but instead apply more to the underdense ones. The purpose of displaying them is discussed in the text. Note that the ordinate shows the midpoints of the height bins; the lowest and highest bins are $4 \mathrm{~km}$ thick, while all others are $3 \mathrm{~km}$ thick.

ever, the match is not perfect. For example, the graphs on the right show large values even in November and December, while Fig. 6a and b do not show excessively negative slopes in those months. Furthermore, when the mean and modal decay times increase, the CCD should shift to the right, pushing the tail to the right, thus resulting in less negative slopes at the chosen decay times. The observed effect is the opposite. Therefore, we do not believe that the effect seen in the slopes is a mathematical artefact related to the overall decay times and conclude that the effect is real. However, whatever mechanisms drive these two parameters (slopes and modes/means) may have a geophysical commonality.

In Fig. 7, the slopes are again more negative at the lowest heights, but are more extremely so at night. Again, this is expected, based on our earlier discussions, and further emphasizes the usefulness of the slope as a diagnostic parameter. Equally importantly, the temporal and spatial variations in the overall means and modes (Fig. 7c and f) do not show any significant similarity (on inverse similarity) to the slopes at the lower heights in the upper plots. Hence, we feel fairly confident that the behaviour of the slopes in the tail of the $\mathrm{CCD}$, and the mean and modal overall decay times, is independent in all cases in Figs. 6 and 7. We will not pursue the reason for annual variations of the decay times in this paper. Note that Lee at al. (2013) have previously proposed a mechanism involving cluster ion chemistry, although some questions remain about the accuracy of some of their reaction rates, especially the high chosen rate of attachment of electrons to aerosols.

It is clear from both Figs. 6 and 7 that the seasonal and day-night differences discussed earlier are real, even when viewed with a larger body of data, and we continue to propose that the effect is related to ozone density variations.

In Fig. 8 we show line plots representing horizontal cuts through the contours in Fig. 7. Representative error bars are also shown; these are hard to evaluate, given the fact that the results are a mixture of instrumental and geophysical variations, some real and some random. Therefore, monthly errors were estimated by fitting a fifth-order polynomial to the monthly data and finding the RMS deviation from this curve. This was taken as a measure of the accuracy of the technique in any month. In most cases the night-time slopes do indeed seem significantly (in a statistical sense) steeper (more negative) than the daytime ones, with the possible exceptions of December in Fig. 8a, May, August, and December in Fig. 8b and April, July, and November in Fig. 8c. Even in these cases, 

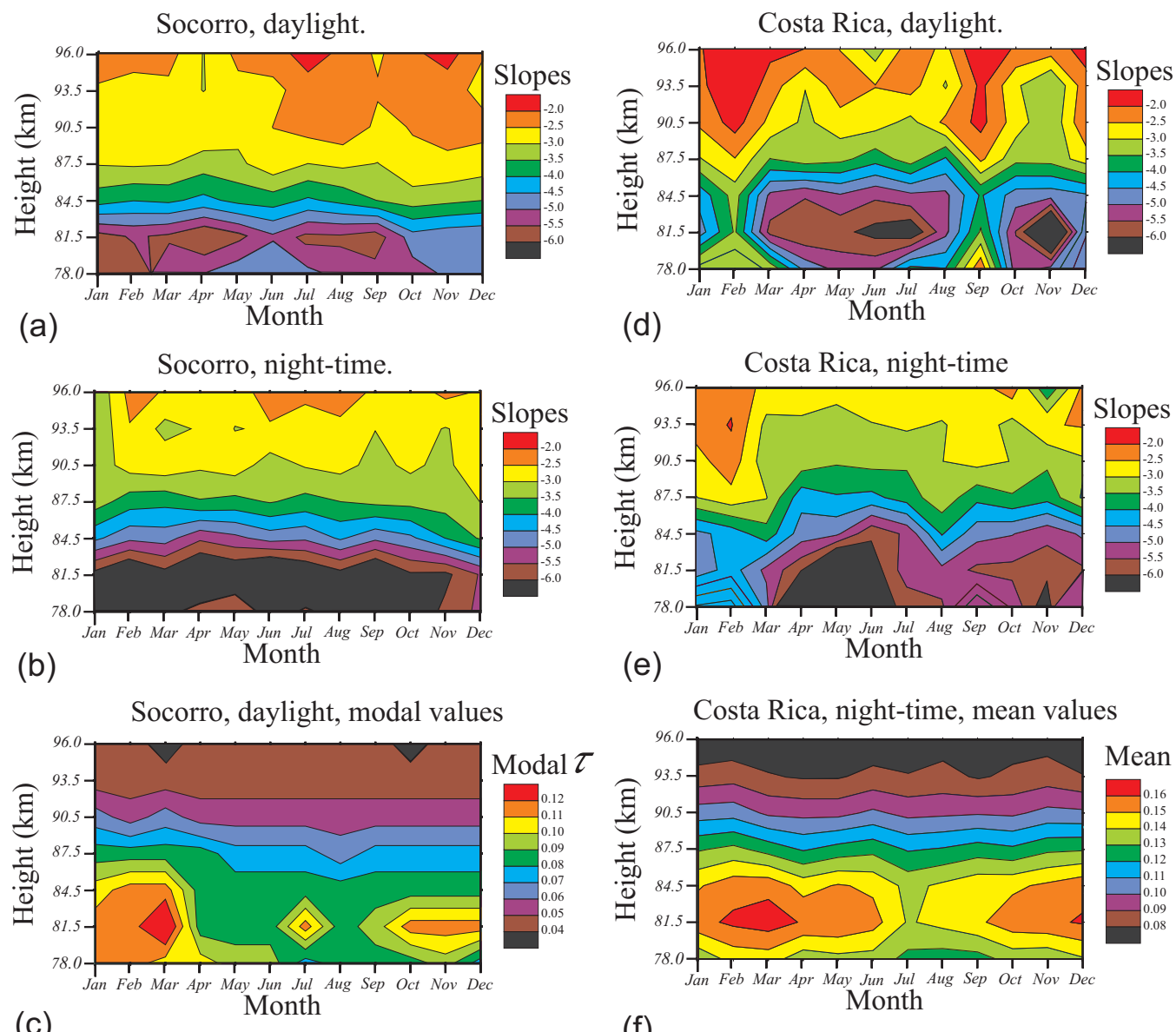

(e) Month

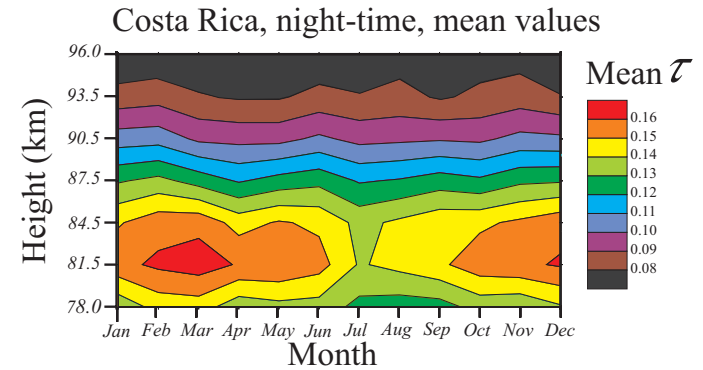

(f)

Figure 7. Panel (a) and (b) show contour plots of the slope of the tail of the CCD, as described in the text, as a function of height and month, for Socorro during daylight hours and night-time respectively. Panel (c) shows contour plots of the modes of $\tau$ during Socorro daylight. Panel (d) and (e) show contour plots of the slope of the tail of the CCD as a function of height and month for Costa Rica during daylight hours and night-time respectively. (f) shows contour plots of the mean values of $\tau$ during Costa Rica night-time. The data below $86 \mathrm{~km}$ in (d) and (e) are discussed in the text. Graphs (c) and (f) do not pertain directly to the transitionally overdense meteors but instead apply more to the underdense ones. The purpose of displaying them is discussed in the text. Note that the ordinate shows the midpoints of the height bins; the lowest and highest bins are $4 \mathrm{~km}$ thick, while all others are $3 \mathrm{~km}$ thick.

however, the night-time slopes are either still more negative than the daytime ones or, in the worst case, equal to them.

It is important to note that although we have concentrated on the lower altitudes (below $86 \mathrm{~km}$ ) in our earlier discussions (mainly because the differences were most visible in the graphs), day-night differences in slopes are evident even up to 89-92 km altitude, and the differences are indeed statistically significant. At the heights of around $90 \mathrm{~km}$, the slopes are less negative than lower down, which may not be unexpected as, for example, ionization efficiencies and reaction rates could vary due to height-dependent constituent and atmospheric densities affecting the slopes. Furthermore, Table $\mathrm{C} 1$ shows that the range of line densities of the trails detected at each height differs - for example, a trail with a line density of $3 \times 10^{15} \mathrm{~m}^{-1}$ would be unlikely to be detected at $80 \mathrm{~km}$ altitude, probably would be detected at $85 \mathrm{~km}$, and very likely would be detected at $90 \mathrm{~km}$ and higher. Thus, each altitude really looks at a mildly different set of meteor types. However, meteor count rates are much higher at $90 \mathrm{~km}$ altitude, so (keeping in mind that the CCDs were normalized to a common "count" of 100000 meteors) there is greater statistical robustness in the fits at $90 \mathrm{~km}$ altitude. Hence, we consider that our results are valid even up to $92 \mathrm{~km}$ altitude.

Figure $8 \mathrm{~d}$ is an interesting case, however. It shows data from the lower heights in Costa Rica. The previous patterns, represented by more negative slopes at night-time, seem to be also applicable during April to July, but the pattern is broken from December to March; in those months, the night-time and daytime slopes are comparable, and in some cases the daytime slopes are even steeper than the night-time ones. Part of a possible resolution of this apparent anomaly can be seen in Fig. 9, where ozone height profile simula- 
(a)
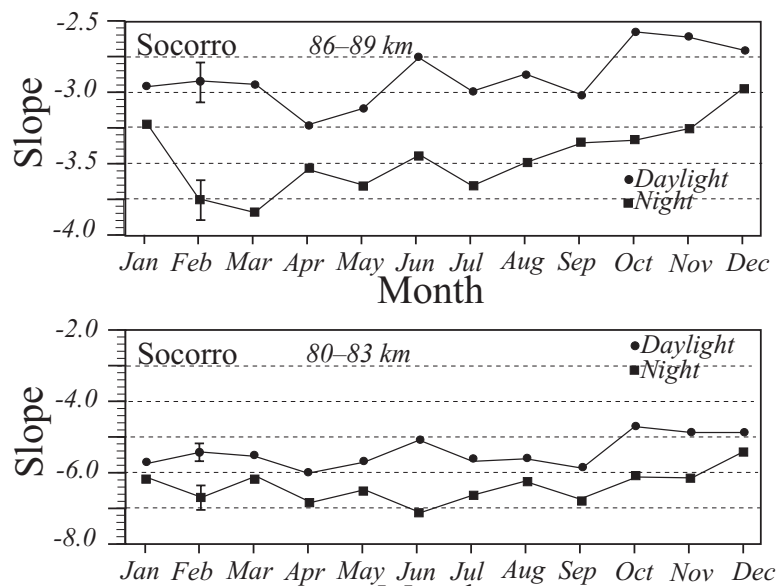

Month

(c)

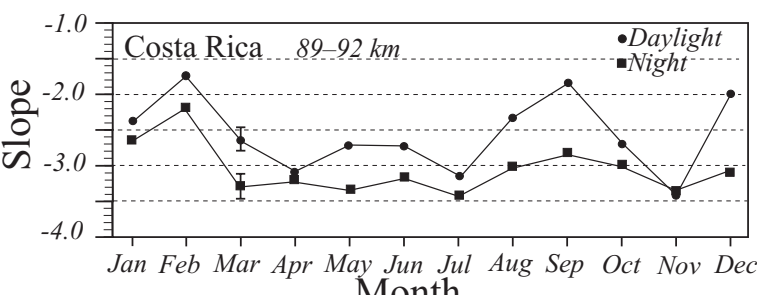
Month

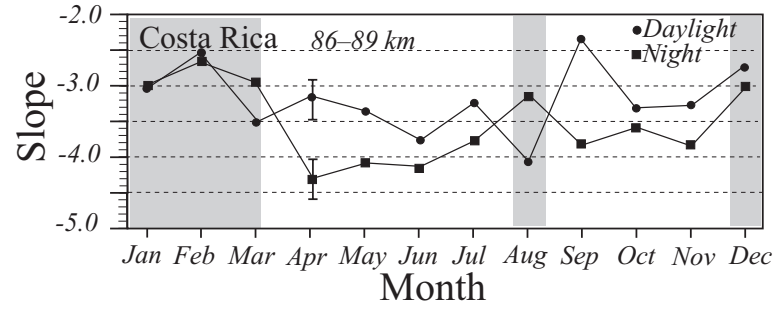

Figure 8. Plots of the slopes of the CCD measured at Socorro and Costa Rica for different selected heights. These plots are representative of all data at the two sites. Representative error bars are shown on each graph. The shading in (d) shows cases where the night-time slopes are similar to, or even less negative than, the daytime ones. These regions are discussed further in the text.

tions from NCAR's Whole Atmosphere Community Climate Model (WACCM4) are shown for Costa Rica and Socorro. It is clear that, in January over Costa Rica, the night-time profile is quite elevated in height, with a peak in concentration at $90 \mathrm{~km}$ altitude and relatively small values at $\sim 83-$ $85 \mathrm{~km}$. The lower-altitude night-time values are even comparable to daytime values at the lower heights (of the order of $0.7 \times 10^{14} \mathrm{~m}^{-3}$ ). Values at Socorro are twice as large as those at Costa Rica at $\sim 83 \mathrm{~km}$, and $50 \%$ larger at $\sim 85 \mathrm{~km}$. Although factors of 1.5 to 2 may not seem large, it needs to be kept in mind that the WACCM data are from a model and may have significant biases, possibly of the order of a factor of 2 or more (as discussed earlier in regard to Fig. 5b), so the possibility exists that the real differences could be even more extreme.

The peak heights could also be mildly in error, and the Costa Rica peak may even be higher than shown. Even an

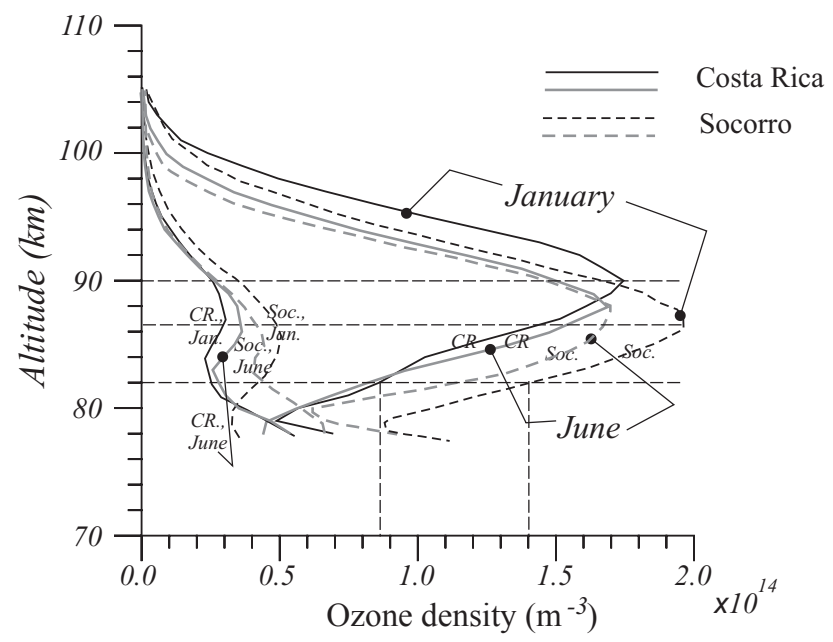

Figure 9. Height profiles of ozone density for Costa Rica and Socorro for January and June, from the WACCM model. Values should only be considered as representative of the real atmosphere to within a factor of typically 2, but shapes of profiles are generally considered representative of reality. Both daytime and night-time values are shown, with the daytime values being much smaller and appearing to the left. Peak heights of ozone density for January nighttime at the two sites are marked as horizontal broken lines, as is the $82 \mathrm{~km}$ altitude level. The increased height of the January peak at Costa Rica relative to Socorro is evident.

increase of only $2 \mathrm{~km}$ would make night-time and daytime densities very similar at $82 \mathrm{~km}$ altitude at the Costa Rica site. Thus, the differences at Costa Rica and Socorro could be even more extreme than indicated by Fig. 9. In any case, the model results do suggest that night-time values at Costa Rica in January at $\sim 82-85 \mathrm{~km}$ could be quite small and lead to slopes comparable to daytime ones. The January results are representative of all the months from December through March, and in these months, the daytime and night-time results are not statistically different. The months from May to August are represented by the June curves in Fig. 9, where the $\sim 85 \mathrm{~km}$ data are more comparable between sites and day-night differences are more evident.

However, this is not the full story. It was noted in Sect. 4 that interference from other radio stations are more common in Costa Rica than at the other sites. Although the false detections are only 4 in 1000, it should be kept in mind that these detections will occur with equal probability at all heights. On the other hand, the meteors have a height distribution peaking at $90 \mathrm{~km}$, i.e. with lower count rates at lower and higher altitudes. Therefore, the interference becomes relatively more important at $80-85 \mathrm{~km}$, where true meteor detections are less frequent than at $90 \mathrm{~km}$. The existence of this interference can considerably distort the slopes in the tail at these lower heights, enhancing the errors. We believe that the steeper daytime slopes in September are an artefact of the interference, and the same is likely to be true even in October (but in 

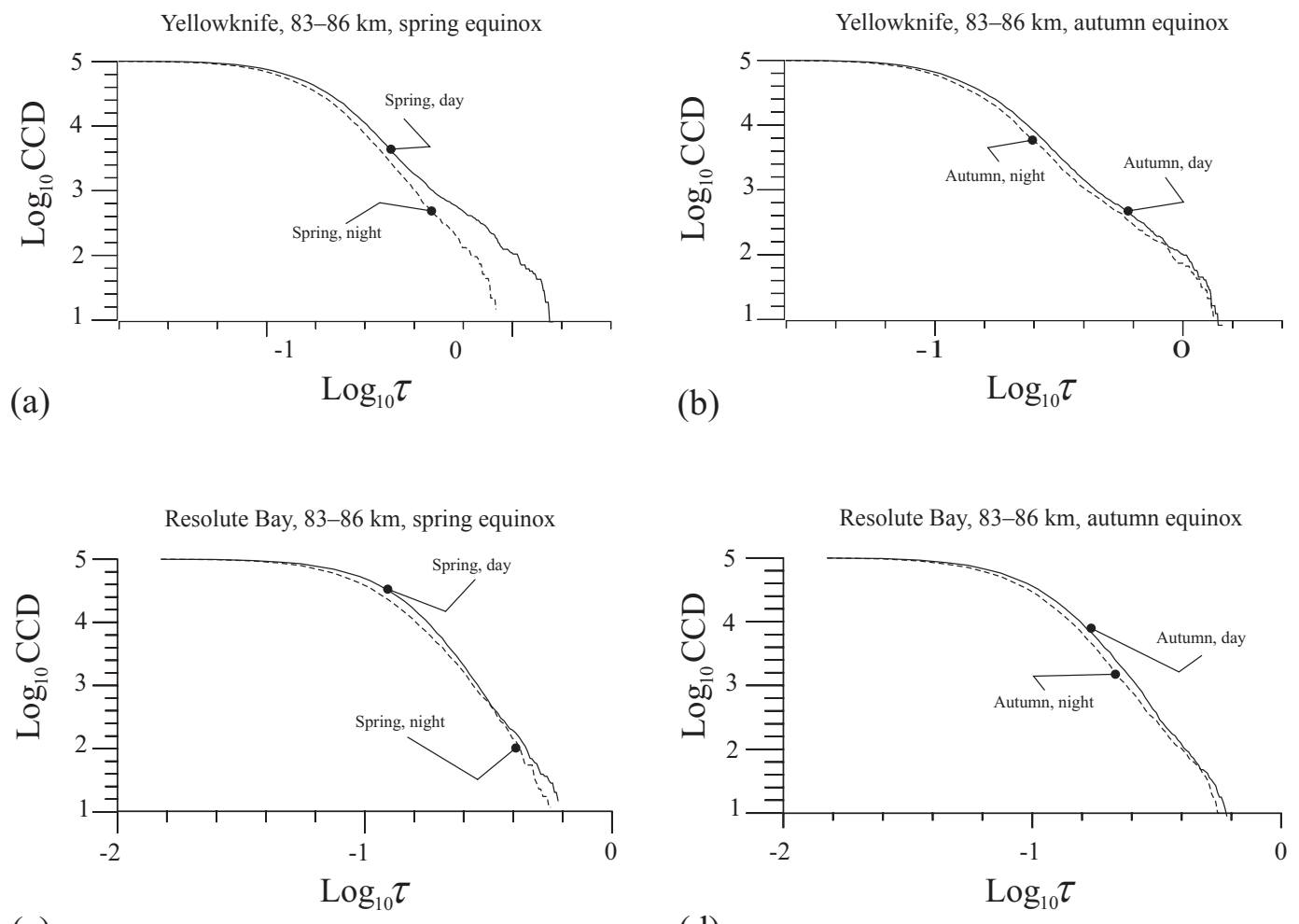

(c)

(d)

Figure 10. $\log _{10}$ CCD graphs (as in Fig. 2) for the two equinoxes at Yellowknife and Resolute Bay for 83-86 km altitude. "Spring equinox" refers to the two months of March and April, while "autumn equinox" refers to the combined results from September and October. Daytime values are shown by solid lines and night-time values by broken lines. Similar results were found for $76-80$ and $80-83 \mathrm{~km}$. Night-time CCD values were always less than daytime ones for $\log _{10} \tau>-1$.

a reverse sense), when the daytime slopes are noticeably less negative than at any other time of the year. We believe that the mixed results at the lower heights in Figs. $7 \mathrm{~b}$ and e and $8 \mathrm{~d}$ are a mixture of the effects discussed above, viz. ozone profiles that are shifted up in height and radio-interference effects.

Nevertheless, the overall picture across all sites is reasonably consistent with an effect on the slopes due to ozone. We have mentioned the exceptions at Costa Rica simply to ensure a complete and unbiased representation of our data, but even these (slightly inconclusive) equatorial results are not at significant variance to our general hypothesis.

We now turn to one final informative graph. In Sect. 5.3, it was noted that during winter and summer, the polar latitude sites are almost entirely in night-time or daylight conditions, so useful day-night comparisons are not possible for much of the year. However, for brief periods around the equinoxes, the polar day has a daylight and a night-time component. We have therefore produced graphs like Fig. 2 for both Yellowknife and Resolute Bay, but concentrating only on the months of March/April (spring equinox) and September/October (autumn equinox). Data were produced for all height ranges shown in Fig. 2, but all showed the same effect, which will now be considered. It should be noted in advance that polar ozone densities are partially controlled by dynamics (especially meridional wind motions), so the impact of strong tides and waves could potentially mean that simple day-night differences seen in Fig. 2 might not be expected here.

Figure 10 shows the results of these determinations. Despite the concern about the possible role of dynamics, it is clear that, at both equinoxes and at both sites, the daytime CCD values at the longer decay times $\left(\log _{10} \tau>-1\right)$ always exceed the night-time ones, just as in Fig. 2. Graphs are only shown for $83-86 \mathrm{~km}$, but results for $80-83$ and $76-80 \mathrm{~km}$ were similar. Indeed, the day-night differences at $80-83 \mathrm{~km}$ were even larger than the $83-86 \mathrm{~km}$ ones. Although the differences in some cases can be slight, it is nevertheless the case that the daytime CCD values for $\log _{10} \tau>-1$ always exceed the night-time ones at both sites, during both equinoctial periods and in all of the height ranges. This is additional evidence that the controlling chemical entity is ozone. Interestingly, the largest day-night difference was in Yellowknife in spring, when a strong springtime ozone maximum has often been seen (see, e.g., Fig. 5a), although that is of secondary importance here. 


\section{Possible physical mechanisms}

With regard to mechanisms, we cannot unambiguously resolve the reasons for the more rapid night-time decays, but we can make some suggestions. Firstly, it is necessary to recall that these are all transitionally overdense meteor trails, with electron line densities generally between about $\sim 2.4 \times 10^{14}$ and $\sim 3.0 \times 10^{15} \mathrm{~m}^{-1}$, with the larger ones being far less common. Secondly, the chemical processes involved do not dominate over diffusion but are a secondary additive process, depleting perhaps $10-30 \%$ of the available electrons. Therefore, at most the chemistry removes maybe $10^{15}$ electrons $\mathrm{m}^{-1}$, and often as low as $10^{14}$. Interestingly, the peak volume densities of the trail for an initial radius of $1 \mathrm{~m}$ are of the order of the line density divided by $\pi$ (Appendix C), or about $10^{14}-10^{15} \mathrm{~m}^{-3}$. Note that these electron volume densities are similar to the night-time ozone densities shown in Fig. 9. Thus, one could speculate that Reactions (R1) and (R2) could be active here, with enough ambient ozone existing to make a measurable impact on electron density depletion. As will be discussed shortly, the ozone is not static but is diffusing into the trail, so as it is depleted, more can be brought in by atmospheric turbulence ("stirred"), further enhancing its availability for reaction.

Nonetheless, some caution is required. First, if there is sufficient atomic O present, then Reaction (R3) could slow the depletion of electrons. The ambient night-time $\mathrm{O}$ concentration around $83 \mathrm{~km}$ is $\sim 10^{17} \mathrm{~m}^{-3}$ (Plane et al., 2015), i.e. the $[\mathrm{O}] /\left[e^{-}\right]$ratio is $100-1000$ in these transitionally overdense trails. However, the ratios of the rate coefficients $k_{3} / k_{2}$ are $4 \times 10^{-5}$ for $\mathrm{FeO}^{+}$and $2 \times 10^{-3}$ for $\mathrm{MgO}^{+}$at mesospheric temperatures (Plane et al., 2015). This means that, in the case of $\mathrm{FeO}^{+}$, the ratio of the reaction rates $k_{3}[\mathrm{O}] / k_{2}\left[e^{-}\right]$is $<0.04$ even at the lowest trail electron line densities $\left(\sim 3 \times 10^{14} \mathrm{~m}^{-1}\right)$. In the case of $\mathrm{MgO}^{+}, \mathrm{Re}-$ action (R2) will be faster than Reaction (R3) at electron densities larger than $2 \times 10^{14} \mathrm{~m}^{-3}$ (equivalent line density $6 \times 10^{14} \mathrm{~m}^{-1}$ ). Therefore, since the $\mathrm{Fe}^{+}$and $\mathrm{Mg}^{+}$densities in the trail should be very similar (Vondrak et al., 2008), the removal rate of electrons should not be significantly slowed down by atomic $\mathrm{O}$ even at the lowest line densities under consideration here.

It also needs to be kept in mind that Reactions (R1) and (R2) are considered to only really become active late in the lifetime of truly overdense trails (after many seconds; e.g. Baggaley, 1979), so why are they active so early here? Furthermore, ozone itself is rapidly destroyed by high temperatures. The time constant for thermal dissociation of $\mathrm{O}_{3}$ at a temperature of $2000 \mathrm{~K}$ and a height of $84 \mathrm{~km}$ can be calculated from the fall-off expression given by Peukert et al. (2013) to be around $10 \mathrm{~ms}$, so why is all the available ozone not quickly destroyed?

In response to the questions in the previous paragraph, the following need to be recognized. First, some of the larger meteors (truly overdense ones, not identified by our radar) may produce strong cylindrical shock fronts, which may have temperatures of the order of $5000 \mathrm{~K}$. Evidence of this was shown with the Leonid meteors by Jenniskens and StenbaekNielsen (2004). However, the issue is still contentious. If such fronts do indeed occur, they will dissociate ozone and even other molecules behind the front, as well as between the front and the trail, and the explosive outward motion may create a barrier to inward infusion of surrounding ambient ozone. This may delay the onset of Reactions (R1) and (R2) for strongly overdense trails (at least until the effect of the shock front has dissipated), consistent with current ideas of delayed onset in the literature.

However, if the transitionally dense trails produce weak or no fronts, this delay may not be significant. It needs to be recognized that a meteor travelling at $40 \mathrm{~km} \mathrm{~s}^{-1}$ will produce a trail $20 \mathrm{~km}$ long over a lifetime of $0.5 \mathrm{~s}$ (although of course the radar will only see the trail in a region of roughly 1 Fresnel zone in width centred around the specular point). If the shock front is weak and is dissipated within $1-2 \mathrm{~km}$ back from the head along the trail, there is still a large region further back where the front has died out and no longer exists (see Appendix B and Fig. B1). Ozone on the trail edge further back may be able to react with the $\mathrm{M}^{+}$ions within the trail via Reaction (R1) much earlier than for overdense meteors. Reaction (R1) is fast with a small temperature dependence for both $\mathrm{Fe}^{+}$and $\mathrm{Mg}^{+}$(Plane et al., 2015), and thus some reactions can occur in the first few milliseconds of the trail, before the ozone is destroyed within the trail and by the front. But if there is only a weak shock front, ambient ozone in the surrounding air can diffuse back into the trail by normal atmospheric diffusion at a later time in the trail evolution. Given that the natural diffusion has a diffusion coefficient (denoted $\kappa$ ) of typically $\kappa \sim 50-100 \mathrm{~m}^{2} \mathrm{~s}^{-1}$ at $90 \mathrm{~km}$ altitude (e.g. Hocking, 1999b), over a timescale of $\Delta t \sim 0.2$ to $0.5 \mathrm{~s}$ (lifetimes of our trails under consideration), ambient ozone may diffuse up to $\Delta x \sim \sqrt{ }(2 \kappa \Delta t)$, or of the order of $10 \mathrm{~m}$. Hence, all the ozone in a cylinder with a final radius of up to $10 \mathrm{~m}$ may be available to react with the trail during its lifetime. As noted above, this "stirring" effect allows the ozone to be more effective in electron removal than simple number-density predictions might suggest.

Electrons may therefore be depleted either in the first 4$8 \mathrm{~ms}$ (maybe $5 \%$ of the available electrons might be depleted at this time) and then again at a later time through reaction with inward-diffusing (turbulently stirred) ambient ozone, after the weak shock (if indeed it exists at all) has been depleted. Possibly up to $10-20 \%$ or more might be depleted in this later time period. As noted, a cylinder with a radius of up to $10 \mathrm{~m}$ is available to supply ozone in the latter case.

On the other hand, the possible existence of stronger shock fronts for strongly overdense trails results in ozone depletion out to radii of many metres transversely all along the trail, protecting the trail from ozone infusion until much later (as also discussed in previous paragraphs, and also see Appendix A). With regard to underdense meteors, they still 
have modestly high temperatures within the trail. Figure 7 in Jenniskens et al. (2004) shows temperatures of the order of 3000-5000 K over a wide range of magnitudes - including underdense. (A meteoroid composition-dependent temperature of approximately $1850 \mathrm{~K}$ is required for onset of a full ablation process, and in fact meteoroids melt at $1850 \mathrm{~K}$, consistent with their olivinic mineral composition; see, e.g., Vondrak et al., 2008.) These initial temperatures of 3000-5000 K will quickly cool to about $2000 \mathrm{~K}$ as the trail expands, so the typical temperature often assumed for underdense meteors is around $1000-2500 \mathrm{~K}$ (we used $2000 \mathrm{~K}$ in our calculation of ozone dissociation times above). Hence, ozone depletion within the first $0.01 \mathrm{~s}$ may occur, but then for further depletion, ambient surrounding ozone needs to infuse. The cylinder of surrounding ambient ozone is much smaller than for longer-lived trails, being $r \sim \sqrt{ }(2 \kappa \Delta t) \sim 4 \mathrm{~m}$ for $\Delta t \sim 0.1 \mathrm{~s}$. Furthermore, Reaction (R1) is dependent on metal ion concentrations, which are of course much lower in underdense trails. Therefore, the impact of $\mathrm{O}_{3}$ may be less evident. The potential effect of Reaction (R3) in reducing the loss rates of electrons should also be recognized, as discussed above.

Thus, it is not unreasonable to consider that the group of meteor trails with line densities between $2.4 \times 10^{14}$ and $3 \times 10^{15} \mathrm{~m}^{-1}$ might be subject to the influence of Reaction (R1), since the lack of a fully developed cylindrical shock front, the existence of comparable ozone and electron/metallic ion densities, a relatively large ratio of electrons to atomic $\mathrm{O}$, and the availability of surrounding ambient ozone diffusing inwards (due to atmospheric turbulence) from a radius of typically 5-10 $\mathrm{m}$ make for conditions favourable to that reaction taking place for this special band of meteor trails.

Our discussions have given a basic model, but future research should allow development of a better one. For example, the importance of shock fronts, and their magnitude as a function of line density, needs to be better quantified. It has been proposed here that many of the reactions occur on the edge of the trail, yet at the same time ozone must diffuse in from outside, producing a mixing process which involves continual replacement of ozone from the surrounding air. The effects of ambient atmospheric diffusion may also not be immediate, being possibly partly overpowered early on by local dynamics within the trail. The relative effectiveness of these processes need to be further studied. In addition, and although not discussed much above, the production of UV radiation during the early stages of trail formation, generated in the shielding hydrodynamic cap in front of the propagating meteor, on the ambient atmosphere should not be discounted (e.g. Sukara, 2014). This effect is well recognized and instrumentally observed. In principle, it is easy to show that the emitted bandwidths of meteor head UV radiation will dissociate some narrow volumetric region of the ambient ozone around the axis of the propagating meteor (see, e.g., Jenniskens and Stenbaeck-Nielsen, 2004). It is then reasonable to expect that the resulting $\mathrm{O}_{2}$ that comes from ozone dissociation (along with the ambient $\mathrm{O}_{2}$ ) will thermally react with the meteor metallic ions on the high-temperature boundaries of the ambipolarly expanding meteor train. The subsequently formed metallic oxide will contribute to electron removal. While the quantifiable contribution of this phenomenon is difficult to determine due to a number of uncertainties, such as optical depth and energy photon density, it should be worth considering in future studies. The magnitude of its effect on early electron removal from the boundaries of the meteor trains should be better identified and constrained. This mechanism will not be limited to only overdense events but will also be significant in strong underdense meteors.

Nevertheless, we believe we have produced a plausible basic mechanism that is consistent with our observations, and that this can form the basis for future developments in this area.

\section{Conclusions}

Evidence has been presented that transitionally dense meteors detected by SKiYMET backscatter meteor radars show increases in the rate of electron loss in the trail at nighttime and at high latitudes during equinoctial conditions. A likely candidate to explain this process seems to be ozone, and both experimental and modelling results support this hypothesis. Results are shown for four radar sites, ranging in latitude from 10 to $75^{\circ} \mathrm{N}$. The chemical processes involved are not dominant but are nevertheless a significant additive effect superimposed upon traditional diffusion. The process is relatively complex, requiring diffusion of ambient ozone into the trail from the surrounding air to replace ozone dissociated by both the initial high temperatures and ozone removal by the excess of metallic ions in the trail, but seems to be a valid mechanism. Further studies are needed to validate this hypothesis, but if the relation can be proven, the possibility exists that meteor backscatter radars may be able to make useful height-dependent measurements of ozone densities on a routine basis (especially bearing in mind that the radars used herein were low power, detecting typically 2000-3000 meteors day ${ }^{-1}$, but higher-power radars already exist which are capable of detection over 20000 meteors day $^{-1}$ ).

\section{Data availability}

Raw meteor data can be accessed on a protected URL by contacting Wayne Hocking at whocking@uwo.ca. The source codes and input data for WACCM model (CESM version 1.1.1 used in this paper) are available from https://svn-ccsm-release.cgd.ucar.edu/model_versions/ cesm1_1_1 upon registration. ERA-Interim data are available through the Meteorological Archival and Retrieval System (MARS) at ECMWF. The WACCM data sets are archived on a University of Leeds networked server for John M. C. Plane's group and available upon request to Plane or Wuhu Feng. 
Appendix A: Underdense and overdense radio-wave scatter
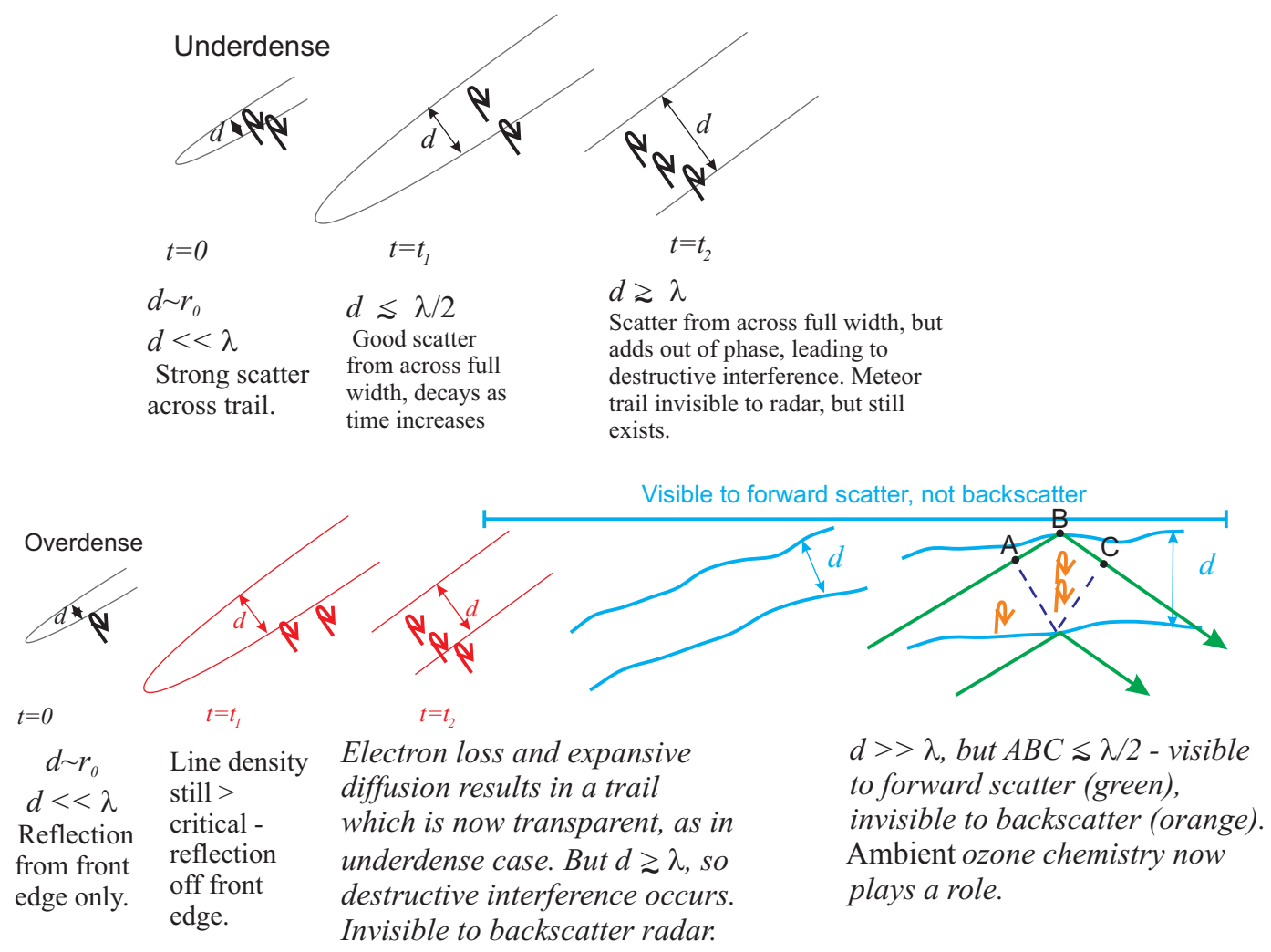

Figure A1. Demonstration of the different geometries associated with backscatter and forward-scatter observation of meteor trails. Note how forward-scatter systems can see the trails to much greater widths than the backscatter case. The upper three panels refer only to underdense backscatter. The lower line of panels shows backscatter behaviour on the left (first three panels), showing the transition to underdense behaviour as time evolves. The two panels on the right show how the tail may twist to become quasi-horizontal, and how the oblique forward scatter allows the tail to be visible to much greater widths than is possible with backscatter. Note that the transition scales indicated $(\lambda / 2, \lambda$ etc.) are approximate. More exact values can be seen in Appendix C. For example, Poulter and Baggaley (1977) suggest that the amplitude of an underdense meteor falls to half its peak when the $1 / e$ density trail radius is $a=0.195 \lambda$, so we might consider a meteor signal as disappearing when the radius is about twice this (since the amplitude is now $1 / 4$ of the peak, and the power is $1 / 16$ of peak power). In this case the $1 / e$ density diameter is $0.78 \lambda$, but the meaning of "disappear" depends on noise levels and initial amplitudes, so this can only be considered as a representative example. The diagrams used here are meant to be applied in a more subjective sense. 


\section{Appendix B: Early stages of meteor trail formation}

This appendix gives a very brief (and simplified) view of meteor trail formation in its early stages and is provided as support for the description given in Sect. 2 of the paper. The main features are drawn in Fig. B1. The structure is extrapolated from several references which discussed flow around solid bodies moving at high speed in the upper atmosphere (e.g. Sarma, 2000). Justification for applying such "hard body" flows is found, for example, in Fig. 1 of Popova et al. (2000), where it is shown that "continuous flow" models have good validity for particles larger than $0.5 \mathrm{~cm}$ at $80 \mathrm{~km}$ altitude and larger than $2 \mathrm{~cm}$ at $90 \mathrm{~km}$ altitude. Further evidence for the validity of this extrapolation can be seen in Jenniskens and Stenbaek-Nielsen (2004), where shock fronts similar to those discussed in Fig. B1 have been observed. Bronshten (1965) also explicitly makes similar assumptions. Other points of support for this assumption are made elsewhere in this appendix. Not all features will be evident in all meteor trails.

In simple terms, as the meteoroid impacts the atmosphere, it collides with atmospheric molecules, and the transfer of kinetic energies results in heating of both particle and the impinging air. The molecules within the meteoroid also boil off once the temperature exceeds $1850 \mathrm{~K}$ (Vondrak et al., 2008). Colliding atmospheric constituents and ablated meteor atoms will form a high-density plasma vapour cloud, as well as an associated dense screening plasma cap in front of the propagating meteor (Popova et al., 2000, 2001). The screening cloud is generally known as the hydrodynamic cap and it is characterized by very high temperatures. It is the onset of this phenomenon that leads to the formation of a first shock front (generally referred to as the ballistic shock front) and thus development of an overall shock envelope that surrounds the axis of meteor propagation. The second shock front, which has a hypersonic-supersonic radial expansion of the envelope parallel to the meteor axis, is known as the cylindrical (bow) shock wave. The characteristics of both the shock front and the bow shock wave are functions of the Knudsen number (value of mean free path divided by meteor size scale), which is generally altered in the case of meteors by the presence of ablation and the hydrodynamic cap (see, e.g., Campbell-Brown and Koschny, 2004).

The vapour cloud is a relatively dense feature, being often orders of magnitude denser than the surrounding air: Popova et al. (2000), at the top of page 113, indicate that the pressure of the vapour cloud can so much exceed the pressure of the surrounding air that it "expands much like a hydrodynamic flow into a vacuum". Hence, the vapour cloud has a very strong presence, further justifying our treatment of it as similar to a bluff solid body and demonstrating the relevance of Fig. B1 to atmospheric flow around a meteoroid.

At this point it is also worth taking another quote from Popova et al. (2000). On page 113, paragraph 3, lines 1-2, those authors state that "when the sonic surface has formed, the flow outside this surface has little effect on the flow near the body". The sonic surface is a surface formed near the outside of the vapour cloud, and in essence this statement says that the flows in the vapour cloud and the external airflow are decoupled; thus, we once again see the vapour cloud acting more as an obstacle, like a solid object, yet again reinforcing the relevance of Fig. B1.

We now return to discussions of the shocks. The bow shock waves are much weaker than the ballistic shock front (Tsikulin, 1970). The latter is capable of completely ionizing and dissociating all atmospheric constituents swept behind the area bound by the ballistic shock front or the hydrodynamic shielding. This ballistic shock region is shown as the thick dark line in front of the meteoroid vapour region in Fig. B1. The size and properties of the ballistic shock will vary, depending primarily on the type of the flow regime but also on the meteoroid size, its material properties (as these impact the rate of ablation), and atmospheric parameters. Depending on the ablation rate and Knudsen number (which are functions of altitude), as well as velocity and the meteor Mach cone, the ballistic shock transitions into the bow shock (as discussed above) at a point denoted as $T_{r}$ in the figure. For small meteoroids (e.g. underdense), the cylindrical shock may not even be produced at higher altitudes.

The cylindrical shock then moves away from the axis at high speed (indicated by the thick grey arrows), where the speed significantly exceeds the local speed of sound. The strength of the bow shock rapidly decays as it moves away from the meteor axis, indicated by the transition from the dark line to the grey broken lines and finally to the weaker dotted lines. In some cases, especially in underdense trails, the cylindrical shock may not exist at all. However, when it does, it has the capacity to dissociate some molecules as it passes through the region denoted as "DI" in the figure, thus affecting the ways in which the remaining molecules, and ions may subsequently interact with the trail. The angle of the bow shock is very close to parallel to the meteor-path axis, often being less than a degree from it. The expression for the angle is $\tan ^{-1}\left(c_{\mathrm{s}} / v_{\mathrm{m}}\right)$, where $c_{\mathrm{s}}$ is the speed perpendicular to the trail axis (usually greater than or equal to the local speed of sound) and $v_{\mathrm{m}}$ is the speed of the meteor.

To complete the picture, the plasma and ablated meteor material entrapped behind the ballistic shock front will enter flow fields behind the meteoroid and will be bottle-necked towards the axis (helped by pressure reductions behind the vapour cloud as a result of flow behaviour). This is a complex process, but in simple terms the air flows back into a region called the "neck" (denoted " $N$ ") where recompression occurs, followed by the development of the short-lasting localized turbulent flows, leading into explosive adiabatic meteor trail formation with some initial radius. More sophisticated understanding of the phenomena is obtained by treating it as an explosive line source (see, e.g., Plooster, 1970; Lin, 1954).

We have not discussed various other features of the process in detail, such as the sonic region, the boundary layer, the stagnation point (source of the UV radiation), the return- 


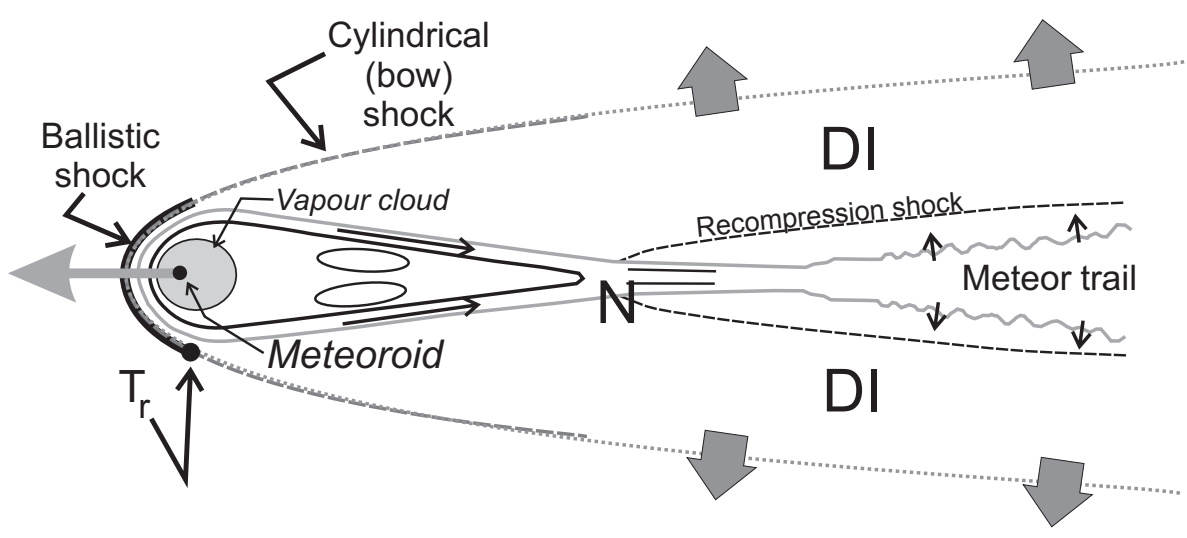

Figure B1. Simplified flow field and shocks expected for transitionally dense and overdense meteors at 80-95 km altitude. The flow fields are based on the flows expected for solid bodies entering the atmosphere (see, e.g., Sarma, 2000, Fig. 49), but with accommodation for differences associated with meteoroid characteristics. Not all features are present in all meteor trails - see the text for details. The figure is not to scale.

flow region, and the "free shear layer", as these are not so important here, but they do add to the complexity of the process. It should also be noted that there are two types of cylindrical shock wave; one is the bow shock discussed above, and the other one is called the recompression (sometimes the vapour) shock wave, which results from recompression of the flow field at the neck " $N$ " (e.g. Bronshten, 1983). The recompression shock front and the cylindrical shock front are almost indistinguishable as they eventually merge into one, shortly outside of the boundary of the adiabatically formed meteor trail with some initial radius.

As noted in the caption, not all features are evident in all trails. In weaker trials, the cylindrical front can be missing, and the "neck" and compressional shock may be absent. However, the vapour cloud, some degree of hydrodynamic shielding, and some form of ballistic shock should be relatively ubiquitous. More details can be found in Hayes and Probstein (1959), Lees and Hromas (1962), Sarma (2000), and Bronshten $(1965,1983)$.

\section{Appendix C: Spatial and temporal scales associated with trails of different line densities}

Most of the theory of backscatter from meteor trails is usually performed using an assumed Gaussian distribution across a cylindrical trail. A key parameter is the line density, or density per unit length. But for reflection purposes, the key parameter is the plasma frequency. First, we will try to better relate the two.

Assuming a volume electron density of the form

$N_{v}=N_{0} \exp \left\{-r^{2} / a^{2}\right\}$

then the line density is found by integrating radially over the whole perpendicular area, i.e.
$N_{\ell}=N_{0} \int_{0}^{\infty} \exp \left\{-r^{2} / a^{2}\right\} 2 \pi r \mathrm{~d} r$.

Using $\zeta=r^{2}$ allows this to be found as

$N_{\ell}=\pi a^{2} N_{0}$.

Let " $a$ " at time $t=0$ be denoted as $r_{0}$, the initial radius. The radius will expand with time according to $a^{2}=r_{0}^{2}+4 D t$, where $D$ is the ambipolar diffusion coefficient.

The critical electron volume density at which total reflection of a radio wave of frequency $f$ is reflected is given by

$N_{v c}=\varepsilon_{0} m_{\mathrm{e}} \omega^{2} /\left(e^{2}\right)=\varepsilon_{0} m_{\mathrm{e}} k^{2} c^{2} /\left(e^{2}\right)$,

where $\omega=2 \pi f, k$ is the wavenumber $(=2 \pi / \lambda, c$ is the speed of light, $m_{e}$ is the mass of an electron, $\varepsilon_{0}$ is the permittivity of free space and $e$ is the electronic charge. Inverting this, we will find the plasma frequencies associated with various line and volume densities. We are especially interested in the peak volume density, since once the peak volume density gets low enough, the trail becomes transparent to the relevant radio wave. The peak volume density is $N_{0}=N_{\ell} /\left(\pi a^{2}\right)$. We will consider the value at $t=0$ as the extreme and take $a$ as the initial trail radius $r_{0}$. This will tell us the frequency required at the trail formation for the trail to appear transparent.

Then

$f_{\text {crit } 0}=e /\left(2 \pi r_{0}\right) \sqrt{ }\left(N_{\ell} / \pi \varepsilon_{0} m_{\mathrm{e}}\right)$.

To calculate $f_{\text {crito }}$, we need $r_{0}$. We will use the following expression for this, from Baggaley and Fisher (1980):

$r_{0}=8.722 \times 10^{-5} \times \exp (0.63 z / 5.75)$, 
Table C1. Various spatial and temporal scales associated with the diffusive expansion of a meteor trail. $f_{\text {crit } 0}$ : critical reflection frequency at

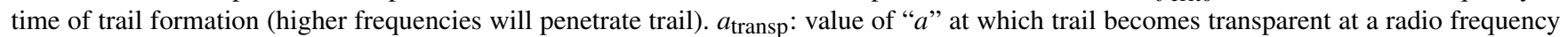
of $35 \mathrm{MHz}(\lambda=8 \mathrm{~m}) . D_{\mathrm{a}}$ : ambient diffusion coefficient. $\tau_{\text {diss }}$ : time to reach " $a_{\text {transp" }}$ " and thus disappear, based on $D_{\mathrm{a}} . D_{\text {plasamb }}$ : geometric

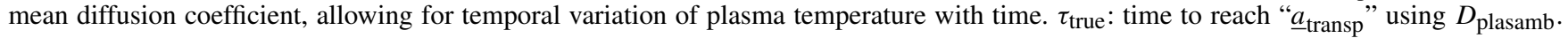
The last group shows the case of line density of $2.4 \times 10^{14}$ electrons $\mathrm{m}^{-1}$, generally regarded as a cut-off density beyond which the trails can no longer be considered as underdense. Bracketed quantities in the last column for the transitional frequency refer to a knee in the CCD of the Socorro radar; these are further discussed in the main text.

\begin{tabular}{|c|c|c|c|c|c|c|c|c|c|}
\hline Line density & $\mathrm{Ht}(\mathrm{km})$ & $r_{0}$ & $f_{\text {crit0 }}$ & $a_{\text {transp }}(\mathrm{m})$ & $a_{\text {transp }} / \lambda$ & $D_{\mathrm{a}}$ & $\tau_{\text {diss }}$ & $D_{\text {plasamb }}\left(\mathrm{m}^{2} \mathrm{~s}^{-1}\right)$ & $\tau_{\text {true }}(\mathrm{s})$ \\
\hline \multirow[t]{5}{*}{$1.0 \times 10^{16}$} & 80 & 0.56 & $904 \mathrm{MHz}$ & 14.5 & \multirow[t]{5}{*}{1.81} & 0.7 & $75 \mathrm{~s}$ & 7 & 7.5 \\
\hline & 85 & 0.97 & $521 \mathrm{MHz}$ & 14.5 & & 2.0 & $26 \mathrm{~s}$ & 20.0 & 2.6 \\
\hline & 90 & 1.67 & $303 \mathrm{MHz}$ & 14.5 & & 4.0 & $13 \mathrm{~s}$ & 40 & 1.3 \\
\hline & 95 & 2.89 & $175 \mathrm{MHz}$ & 14.5 & & 8.0 & $6.4 \mathrm{~s}$ & 80 & 0.64 \\
\hline & 100 & 5.0 & $101 \mathrm{MHz}$ & 14.5 & & 20.0 & $2.3 \mathrm{~s}$ & 200 & 0.23 \\
\hline \multirow[t]{5}{*}{$3.0 \times 10^{15}$} & 80 & 0.56 & $495 \mathrm{MHz}$ & 7.9 & \multirow[t]{5}{*}{0.99} & 0.7 & $22.3 \mathrm{~s}$ & 5.5 & 2.8 \\
\hline & 85 & 0.97 & $286 \mathrm{MHz}$ & 7.9 & & 2.0 & $7.7 \mathrm{~s}$ & 16 & 0.96 \\
\hline & 90 & 1.67 & $166 \mathrm{MHz}$ & 7.9 & & 4.0 & $3.7 \mathrm{~s}$ & 32 & 0.46 \\
\hline & 95 & 2.89 & $96 \mathrm{MHz}$ & 7.9 & & 8.0 & $1.7 \mathrm{~s}$ & 64 & 0.11 \\
\hline & 100 & 5.0 & $55.5 \mathrm{MHz}$ & 7.9 & & 20.0 & $0.47 \mathrm{~s}$ & 160 & 0.06 \\
\hline \multirow[t]{5}{*}{$1.0 \times 10^{15}$} & 80 & 0.56 & $286 \mathrm{MHz}$ & 4.6 & \multirow[t]{5}{*}{0.58} & 0.7 & $7.4 \mathrm{~s}$ & 4.2 & 1.23 \\
\hline & 85 & 0.97 & $165 \mathrm{MHz}$ & 4.6 & & 2.0 & $2.5 \mathrm{~s}$ & 12 & 0.42 \\
\hline & 90 & 1.67 & $96 \mathrm{MHz}$ & 4.6 & & 4.0 & $1.2 \mathrm{~s}$ & 24 & 0.2 \\
\hline & 95 & 2.89 & $55.4 \mathrm{MHz}$ & 4.6 & & 8.0 & $0.4 \mathrm{~s}$ & 48 & 0.07 \\
\hline & 100 & 5.0 & $32.0 \mathrm{MHz}$ & 4.6 & & 20.0 & - & 120 & - \\
\hline \multirow[t]{5}{*}{$2.4 \times 10^{14}$} & 80 & 0.56 & $140 \mathrm{MHz}$ & 2.24 & \multirow[t]{5}{*}{0.28} & 0.7 & $1.7 \mathrm{~s}$ & 2.8 & $0.42(0.2)$ \\
\hline & 85 & 0.97 & $89 \mathrm{MHz}$ & 2.24 & & 2.0 & $0.51 \mathrm{~s}$ & 8 & $0.13(0.10)$ \\
\hline & 90 & 1.67 & $47.0 \mathrm{MHz}$ & 2.24 & & 4.0 & $0.14 \mathrm{~s}$ & 16 & $0.035(0.06)$ \\
\hline & 95 & 2.89 & $27.1 \mathrm{MHz}$ & 2.24 & & 8.0 & - & 32 & - \\
\hline & 100 & 5.0 & $15.7 \mathrm{MHz}$ & 2.24 & & 20.0 & - & 80 & - \\
\hline
\end{tabular}

where $z$ is in kilometres above sea level.

For radar frequencies above $f_{\text {crito }}$, the incident wave will not reach a point of zero refractive index and may pass through the trail, leading to partial reflections within the trail from various points, and potential self-cancellation (i.e. the scatter is essentially an underdense case).

We next ask the following questions: what does the radius " $a$ " have to be to reach a density at which a $35 \mathrm{MHz}$ radio wave will be able to just pass through the trail, and how long will it take to reach this value of " $a$ "? (we choose a value of $35 \mathrm{MHz}$ since it is representative of the frequencies of most of our radars). We will call this $a_{\text {transp }}$, since at this radius the trail becomes transparent.

This requires that

$N_{0}=N_{\ell} /\left(\pi a_{\text {transp }}^{2}\right)=\varepsilon_{0} m_{\mathrm{e}} \omega^{2} /\left(e^{2}\right)$

where $N_{0}$ is the peak volume electron density.

Hence,

$a_{\text {transp }}=e /(2 \pi f) \sqrt{ }\left(N_{\ell} /\left(\varepsilon_{0} \pi m_{\mathrm{e}}\right)\right)$,

where $f$ is the frequency of interest (here, $35 \mathrm{MHz}$ ). These values are shown in the fifth column of the table above.
The quantity $a_{\text {transp }} / \lambda$ is shown as the sixth column. From Poulter and Baggeley (1978, Fig. 1), an underdense echo falls to half of its original amplitude at $(k a)^{2} \sim 1.5$, or $(2 \pi a / \lambda) \sim \sqrt{ }(1.5)$, or

$a / \lambda \sim 0.195$.

Therefore, if $a / \lambda$ in the table substantially exceeds 0.2 , the meteor trail will disappear from the radar screen as soon as it becomes underdense. In the table, only the last case is close to this - such a meteor will start as "overdense", i.e. will not show a decrease in amplitude with time, and then will reach semi-transparency and show a weak exponential decay in its tail. All other cases in the table will expand with no decrease in reflected amplitude (the meteor amplitude looks flat, though perhaps may have some wobbles due to turbulence and maybe polarization effects; Baggeley and Fisher, 1980, Fig. 2) and then reach underdense status and abruptly disappear, since the trail $1 / e$ half-width is much larger than $0.2 \lambda$ at this point.

Our final question is, how long will it take for the trail to reach the point where it becomes marginally underdense? This will define the longest; possible visual lifetime for trails of this line density. Here we use the relation 
$a^{2}=r_{0}^{2}+4 D t$,

(since the trail expands according to $N_{0} \exp \left\{-r^{2} / a^{2}\right\}=$ $\left.n(r, t)=\frac{N_{l}}{\pi\left(r_{0}^{2}+4 D t\right)} \exp \left[\frac{-\left(r^{2}\right)}{r_{0}^{2}+4 D t}\right]\right)$, so we need to determine $D$. The term out the front of the "exp" term on the right hand side is a normalization which keeps the total number of electrons in the trail conserved - we have called it $N_{\ell} /\left(\pi a^{2}\right)$ in our equations above. We do this using Jones and Jones (1990).

Then

$t=\left(a^{2}-r_{0}^{2}\right) /(4 D)$.

Our values of $D$ were read from the graph of Jones and Jones (1990) Fig. 2. Times are tabulated in the last column. Some are negative, probably indicating that $r_{0}$ is too large for these lower line densities. These are of no real concern to us - a smaller value of $r_{0}$ would allow them to be positive, and $r_{0}$ can have considerable variability about the value given by the formula above.

Some warnings are necessary here. First, we note that, in all of our calculations, we assume that the expansion of the trail is driven by molecular diffusion. However, the background atmosphere has a natural diffusion rate of its own due to turbulence, with a diffusion coefficient of up to $100 \mathrm{~m}^{2} \mathrm{~s}^{-1}$, and eventually this will come into play, though it will take at least a few tenths of a second or more. Secondly, since the software determines the decay times by using times for the auto- and cross-covariance to fall to one-half of the peak value, the meaning of the "half-life" can be a little different for underdense and non-underdense cases. The underdense cases will have an exponential decay, while the more dense trails will in essence have a boxcar format, so the difference in waveform shape can affect the meaning of the "decay time". For the decay times discussed here, it really only matters that we are consistent in our definitions of decay time for any class of meteor trail, but the differences should be recognized.

Returning to the issue of trail lifetime, Greenhow (1952) introduced the follow expression to determine the lifetime:

$\tau=r_{\mathrm{e}} /\left(4 \pi^{2}\right) N_{\ell} \lambda^{2} / D_{\mathrm{a}}$,

where $r_{\mathrm{e}}$ is the electron radius $\left(2.8 \times 10^{-15}\right)$ and $D_{\mathrm{a}}$ is the ambient diffusion coefficient. This is essentially derived in the same way that we have developed above, except that $r_{0}$ was set to zero. For $N_{\ell}=1.0 \times 10^{16}$ and $\lambda=8 \mathrm{~m}$, this is gives $\tau=45.4 / D_{\mathrm{a}}-$ so in Table $\mathrm{C} 1$, at $80 \mathrm{~km}$ with $D_{\mathrm{a}}=0.7$, this gives $65 \mathrm{~s}$, compared with the estimate of $75 \mathrm{~s}$ given there. Hence, it is consistent.

However, use of $D_{\mathrm{a}}$ in this way, although having been done in the past, seems to be in error. In truth we have a small number of high-speed ions (part of the plasma) diffusing through a more dense background in which the molecules are moving much more slowly. To begin, consider that the charge on the ion is of little consequence, and that it bounces off hard, billiard-ball targets in the form of neutral atoms. This is a simple diffusion problem encountered in many elementary university textbooks (e.g. Tabor, 1969). The result is a diffusion coefficient given by

$D=1 / 3 c_{\mathrm{RMS}} \ell_{\mathrm{p}}$.

where $c_{\mathrm{RMS}}$ is the root-mean-square speed of the diffusing molecule (or ion in this case) and $\ell_{\mathrm{p}}$ is the mean free path in the background atmosphere. Here, we may write $c_{\mathrm{RMS}}=$ $\sqrt{ }\left(3 k_{\mathrm{B}} T_{\mathrm{p}} / m_{\mathrm{i}}\right)$, where $T_{\mathrm{p}}$ is the temperature of the diffusing plasma, $k_{\mathrm{B}}$ is Boltzmann's constant, and $m_{\mathrm{i}}$ is the ion mass. We assume that the electrons are dragged along by the heavier ions, but the most important point is the dependence on the square root of $T_{\mathrm{p}}$. However, while $c_{\mathrm{RMS}}$ depends on the square root of the plasma ion temperature, the mean free path is given by $\ell_{\mathrm{p}}$, which is proportional to $1 /\left(\sigma^{2} n\right)$, where $\sigma$ is the mean molecular diameter of the ion and the neutrals and $n$ is the neutral number density of particles. The important point is the dependence on $n$, which is proportional to $P / T_{\mathrm{a}}$, with $P$ being the pressure of the gas and $T_{\mathrm{a}}$ being the ambient gas temperature. Hence, we may write that the diffusion rate of the ion through the neutral gas is

$D_{\mathrm{pa}} \propto T_{\mathrm{p}}^{1 / 2} T_{\mathrm{a}} / P$.

In the case of a real ion, Coulomb forces exist between the ion and the electrons of the scattering molecules, giving an effective value of $\sigma$ which is weakly dependent on the ion speed, since depths of penetration during collisions may depend on speed. The scaling constant in the term $\ell_{\mathrm{p}} \propto 1 /\left(\sigma^{2} n\right)$ also changes, being dependent on the relative speed of the ions and the neutrals. The net result is that the term $T_{\mathrm{p}}^{1 / 2}$ in the expression for $D_{\mathrm{pa}}$ for becomes a power of 1 , viz.

$D_{\text {pa }} \propto T_{\mathrm{p}} T_{\mathrm{a}} / P$.

This is often written as $T^{2} / P$, but this is only valid when $T_{\mathrm{p}}$ and $T_{\mathrm{a}}$ are the same. In the more general case, the diffusion coefficient is given by the above expression.

Another way to consider this is to use $D_{\mathrm{pa}}$ as the geometric mean of the ambient diffusion rate and the plasma diffusion rate.

This means that the diffusion coefficients in column 7 of Table $\mathrm{C} 1$ must be rescaled by $T_{\mathrm{p}} / T_{\mathrm{a}}$. This is an important point which we must now incorporate. If the plasma temperature is $5000 \mathrm{~K}$, and the ambient temperature is $250 \mathrm{~K}$, the rescaling is a factor of 20 . Additional terms may need to be considered, such as the different masses and radii of the ions relative to the neutrals, but these are less significant re-scalings. It should be noted that not all neutral molecules will be at the ambient temperature - those that suffered collisions with metal ions during the meteor entry will of course 
be hot, but they will be similar in number to the ion density and thus many thousands of times fewer in number than the ambient neutrals. Successive collisions of the heated neutrals and ions with the ambient molecules will of course drive the whole system to an equilibrium temperature, but such an equilibrium occurs later and cannot be assumed yet.

However, we also need to recognize one more feature, namely that the plasma rapidly cools as it spreads. As it cools, the effective plasma diffusion coefficient also decreases, so the diffusion becomes less rapid. At the same time the neutral gas also becomes hotter, as the two species attempt to develop thermal equilibrium. The exact interaction is complex, though one can get some idea of the rate of transfer by assuming total heat content is conserved as the trail expands, so the temperature must fall off proportionally to the area of cross section of the trail. A detailed treatment of the evolution toward equilibrium is beyond the scope of this appendix: we will assume that the effective diffusion coefficient can be found as the geometric mean of the ambient and plasma diffusion rates and will then divide by a further factor of 2 to attempt to cover the slower diffusion later on.

Then we replace the diffusion coefficient in column 7 of Table C1 by $D_{\mathrm{pa}}=(1 / 2) D_{\mathrm{a}} T_{\mathrm{p}} / T_{\mathrm{a}}$, which is given in column 9. We will use $T_{\mathrm{p}}=5000 \mathrm{~K}$ for a line density of $1.0 \times 10^{16}, 4000 \mathrm{~K}$ for a line density of $3.0 \times 10^{15}, 3000 \mathrm{~K}$ for a line density of $1.0 \times 10^{15}$, and $2000 \mathrm{~K}$ for a line density of $2.4 \times 10^{14}$. These are guesses based partly on estimates of a max temperature of $5000 \mathrm{~K}$ for an overdense meteor (Jenniskens and Stenbaek-Nielsen, 2004). An ambient temperature of $250 \mathrm{~K}$ will be assumed. $D_{\mathrm{pa}}$ is denoted as $D_{\text {plasamb in }}$ column 9. Diffusion timescales are then deduced in the same way as those given in column 8 , but using the improved diffusion coefficients, and are shown in column 10.

The results shown in column 10 help us determine the meteors that the radar can detect. Meteors with timescales larger than $2 \mathrm{~s}$ will not be seen by the SKiYMET radars. Hence, meteors with line densities in excess of $1.0 \times 10^{16}{\text { electrons } \mathrm{m}^{-1}}^{-1}$ will not be seen below $85 \mathrm{~km}$ altitude, and if even more dense then probably not below $90 \mathrm{~km}$. Line densities below $3.0 \times 10^{15}$ will not be seen below $85 \mathrm{~km}$.

We may therefore conclude that the vast majority of the "larger" meteors seen in our studies will not be overdense but will probably be transitional. Of course, the weaker ones will all be underdense. However, we will rarely see truly overdense meteors in our studies. This is an important point. 
Acknowledgements. We thank Paul Krehbiel, Ron Thomas, and their staff, as well as Alan Erickson, for support of the Socorro radar site; George Jensen and Ross Ashlie for assistance with the Yellowknife radar; and the staff of Narwhal and ATCO (especially Rick and Byron Gaulton) and SRI (especially John Kelly, Craig Heiselman, Mike Nicholls) for support at the Resolute Bay site. We are also grateful to Anne Smith (NCAR) for supplying satellite data from Smith et al. (2013). R. E. Silber acknowledges an NSERC CREATE Training Program for Integrating Atmospheric Chemistry and Physics from Earth to Space (IACPES) and a Northern Scientific Training Program grant. We also acknowledge support from the Natural Sciences and Engineering Research Council of Canada. J. M. C. Plane and W. Feng were supported by the European Research Council (project number 291332 - CODITA). Useful discussions with Peter Brown and Iain Boyd are recognized, and the support of the University of Costa Rica for the meteor radar site in Santa Cruz is acknowledged.

The topical editor, C. Jacobi, thanks two anonymous referees for help in evaluating this paper.

\section{References}

Anderson, Jr., J. D.: Hypersonic and high-temperature gas dynamics , 2nd Edn., AIAA ("American Institute of Aeronautics and Astronautics” Inc.), Reston, VA, USA, 813 pp., 2006.

Baggaley, W. J.: The de-ionization of dense meteor trains, Planet. Space Sci., 26, 979-981, doi:10.1016/0032-0633(78)90080-6, 1978.

Baggaley, W. J.: The interpretation of overdense radio meteor echo duration characteristics, B. Astron. I. Czech., 30, 184-189, 1979.

Baggaley, W. J. and Fisher, G. W.: Measurements of the initial radii of the ionization columns of bright meteors, Planet. Space Sci., 28, 6, 575-580, doi:10.1016/0032-0633(80)90002-1, 1980

Berezhnoy, A. A. and Borovička, J.: Formation of molecules in bright meteors, Icarus, 210, 150-157, doi:10.1016/j.icarus.2010.06.036, 2010.

Bones, D., Plane, J. M. C., and Feng, W.: Dissociative Recombination of $\mathrm{FeO}^{+}$with Electrons: Implications for Plasma Layers in the Ionosphere, J. Phys. Chem. A, 120, 1369-1376, 2016.

Boyd, I. D.: Computation of Atmospheric Entry Flow about a Leonid Meteoroid, Earth Moon Planets, 82, 93-108, doi:10.1007/978-94-017-2071-7_8, 2000.

Bronshten, V. A.: Problems of the movements of large meteoric bodies in the atmosphere, United States Air force Project Rand, Translated by Gazeley, J. B., edited by: Romig, M. F., RM-4257PR, 1965.

Bronshten, V. A.: Physics of meteoric phenomena, Fizikameteornykhiavlenii, Moscow, Izdatel'stvoNauka, 1981, D. Reidel Publishing Co., Dordrecht, the Netherlands, 1983.

Campbell-Brown, M. D. and Koschny, D.: Model of the ablation of faint meteors, Astron. Astrophys., 418, 2, 751-758, doi:10.1051/0004-6361:20041001, 2004.

Ceplecha, Z., Borovička, J., Elford, W. G., ReVelle, D. O., Hawkes, R. L., Porubčan, V., and Šimek, M.: Meteor phenomena and bodies, Space Sci. Rev., 84, 327-471, doi:10.1023/A:1005069928850, 1998.
Cevolani, G. and Pupillo, G.: Ground-based radio observations to probe the ozone content in the meteor region, Ann. Geophys.Italy, 46, 247-258, doi:10.4401/ag-3399, 2003.

Cevolani, G., Hajduk, A., Hajdukova, M., Porubčan, V., and Trivellone, G.: Ozone concentration at meteor heights determined from forward-scatter radar echoes, J. Atmos. Sol.-Terr. Phy., 61, 539543, doi:10.1016/S1364-6826(99)00019-X, 1999.

Dee, D. P., Uppala, S. M., Simmons, A. J., Berrisford, P., Poli, P., Kobayashi, S., Andrae, U., Balmaseda, M. A., Balsamo, G., Bauer, P., Bechtold, P., Beljaars, A. C. M., van de Berg, L., Bidlot, J., Bormann, N., Delsol, C., Dragani, R., Fuentes, M., Geer, A. J., Haimberger, L., Healy, S. B., Hersbach, H., Hólm, E. V., Isaksen, L., Kållberg, P., Köhler, M., Matricardi, M., McNally, A. P., Monge-Sanz, B. M., Morcrette, J.-J., Park, B.-K., Peubey, C., de Rosnay, P., Tavolato, C., Thépaut, J.-N., and Vitart, F., The ERA-Interim reanalysis: Configuration and performance of the data assimilation system, Q. J. Roy. Meteor. Soc., 137, 553-597, doi:10.1002/qj.828, 2011.

Garcia, R. R. and Solomon, S.: A Numerical Model of the Zonally Averaged Dynamical and Chemical Structure of the Middle Atmosphere, J. Geophys. Res., 88, 1379-1400, 1983.

Greenhow, J. S.: Characteristics of radio echoes from meteor trails: III, The behaviour of the electron trails after formation, P. Phys. Soc. B, 65, 169-181, doi:10.1088/0370-1301/65/3/301, 1952.

Hayes, W. D. and Probstein, R. F.: Hypersonic Flow Theory (Applied Mathematics and Mechanics), 464 pp., Academic Press, New York, USA, 1959.

Hocking, W. K.: Temperatures using radar-meteor decay times, Geophys. Res. Letts., 26, 3297-3300, 1999a.

Hocking, W. K.: The Dynamical Parameters of Turbulence Theory as they apply to Middle Atmosphere Studies, Earth Planets Space, 51, 525-541, 1999 b.

Hocking, W. K., Fuller, B., and Vandepeer, B.: Real-time determination of meteor-related parameters utilizing modern digital technology, J. Atmos. Sol.-Terr. Phy., 63, 155-169, doi:10.1016/S1364-6826(00)00138-3, 2001.

Jenniskens, P. and Stenbaek-Nielsen, H. C.: Meteor wake in high frame-rate images-implications for the chemistry of ablated organic compounds, Astrobiology, 4, 95-108, doi:10.1089/153110704773600267, 2004.

Jenniskens, P., Laux, C. O., Wilson, M. A., and Schaller, E. L.: The mass and speed dependence of meteor air plasma temperatures, Astrobiology, 4, 81-94, doi:10.1089/153110704773600258, 2004.

Jenniskens, P., Wilson, M. A., Packan, D., Laux, C. O., Krüger, C. H., Boyd, I. D., Popova, O. P., and Fonda, M.: Meteors: A delivery mechanism of organic matter to the early Earth, Leonid Storm Research, Springer Netherlands, 57-70, doi:10.1007/97894-017-2071-7_5, 2000.

Jones, J. and Campbell-Brown, M.: The initial train radius of sporadic meteors, Mon. Not. R. Astron. Soc., 359, 1131-1136, doi:10.1111/j.1365-2966.2005.08972.x, 2005.

Jones, W. and Jones, J.: Ionic diffusion in meteor trains, J. Atmos. Sol.-Terr. Phy., 52, 185-191, doi:10.1016/0021-9169(90)901224, 1990.

Jones, J. and Simek, M.: A note on the determination of ozone concentrations from radio-meteor duration distributions, Earth Moon Planets, 68, 329-338, 1995. 
Jones, J., McIntosh, B. A., and Simek, M.: Ozone and the duration of overdense radio meteors, J. Atmos. Sol-Terr. Phy., 52, 253258, doi:10.1016/0021-9169(90)90092-2, 1990.

Jones, W.: Theory of the initial radius of meteor trains, Mon. Not. R. Astron. Soc., 275, 812-818, doi:10.1093/mnras/275.3.812, 1995.

Josyula, E. and Burt J.: Review of Rarefied Gas Effects in Hypersonic Applications, DTIC Document, Air Force Research Lab Wright-Patterson AFB OH, 2011.

Kaiser, T. R.: Radio echo studies of meteor ionization, Adv. Phys., 2, 495-544, doi:10.1080/00018735300101282, 1953.

Lee, C. S., Younger, J. P., Reid, I. M., Kim, Y. H., and Kim, J. H.: The effect of recombination and attachment on meteor radar diffusion coefficient profiles, J. Geophys. Res.-Atmos., 118, 30373043, doi:10.1002/jgrd.50315, 2013.

Lees, L. and Hromas, L.: Turbulent Diffusion in the Wake of a Blunt-Nosed Body at Hypersonic Speeds, J. Aerosp. Sci., 29, 976-993, doi:10.2514/8.9671, 1962.

Lin, S. C.: Cylindrical shock waves produced by instantaneous energy release, J. Appl. Phys., 25, 54-57, doi:10.1063/1.1721520doi:10.2514/8.9671, 1954.

Marsh, D. R., Mills, M. J., Kinnison, D. E., Lamarque, J.-F., Calvo, N., and Polvani, L. M.: Climate Change from 1850 to 2005 Simulated in CESM1(WACCM), J. Climate, 26, 7372-7391, doi:10.1175/JCLI-D-12-00558.1, 2013.

McKinley, D. W. R.: Meteor science and engineering, McGrawHill, New York, USA, 1961.

McNeil, W. J., Lai, S. T., and Murad, E.: Differential ablation of cosmic dust and implications for the relative abundances of atmospheric metals, J. Geophys. Res.-Atmos., 103, 10899-10911, doi:10.1029/98JD00282, 1998.

McNiel, W. J., Murad, E., and Plane, J. M. C.: Models of Meteoric metals in the Atmosphere, in: Meteors in the Earth's Atmosphere: Meteoroids and Cosmic dust and their interactions with the Earth's Upper Atmosphere, edited by: Murad, E. and Williams, I. P., 265-288, Cambridge University Press, Cambridge, New York, USA, 323 pp., 2002.

Menees, G. P. and Park, C.: Nitric oxide formation by meteoroids in the upper atmosphere, Atmos. Environ., 10, 535-545, doi:10.1016/0004-6981(76)90180-3, 1976.

Öpik, E. J.: Physics of meteor flight in the atmosphere, Interscience Publishers, New York, USA, 1958.

Park, C. and Menees, G. P.: Odd nitrogen production by meteoroids, J. Geophys. Res.-Oceans, 83, C8, 4029-4035, doi:10.1029/JC083iC08p04029, 1978.

Plane, J. M.: Atmospheric chemistry of meteoric metals, Chem. Rev., 103, 4963-4984, doi:10.1021/cr0205309, 2003.

Plane, J. M.: Cosmic dust in the earth's atmosphere, Chem. Soc. Rev., 41, 6507-6518, doi:10.1039/C2CS35132C, 2012.

Plane, J. M. C., Feng, W., and Dawkins, E. C. M., The Mesosphere and Metals: Chemistry and Changes, Chem. Rev., 115, 44974541, doi:10.1021/cr500501m, 2015.

Plane, J. M. C., Gómez-Martín, J. C., Feng, W., and Janches, D.: Silicon chemistry in the mesosphere and lower thermosphere, J. Geophys. Res.-Atmos., 121, 3718-3728, doi:10.1002/2015JD024691, 2016.

Plooster, M. N.: Shock waves from line sources. Numerical solutions and experimental measurements, Phys. Fluids, 13, 2665$2675,1970$.
Popova, O. P., Sidneva, S. N., Shuvalov, V. V., and Strelkov, A. S.: Screening of meteoroids by ablation vapor in high-velocity meteors, Earth Moon Planets, 82, 109-128, doi:10.1023/A:1017063007210, 2000.

Popova, O. P., Sidneva, S. N., Strelkov, A. S., and Shuvalov, V. V.: Formation of disturbed area around fast meteor body, Meteoroids 2001 Conference, 495, 237-245, doi:10.1023/A:1017063007210, 2001.

Poulter, E. M. and Baggaley, W. I.: The applications of radio-wave scattering theory to radio-meteor observations, Planet. Space Sci., 26, 969-977, doi:10.1016/0032-0633(78)90079-X, 1978.

Poulter, E. M. and Baggaley, W. J.: Radiowave scattering from meteoric ionization, J. Atmos. Sol-Terr. Phys., 39, 757-768, doi:10.1016/0021-9169(77)90137-4, 1977.

Peukert, S. L., Sivaramakrishnan, R., and Michael, J. V.: High Temperature Shock Tube Studies on the Thermal Decomposition of $\mathrm{O}_{3}$ and the Reaction of Dimethyl Carbonate with O-Atoms, J. Phys. Chem. A, 117, 3729-3738, 2013.

ReVelle, D. O.: Acoustics of meteors-effects of the atmospheric temperature and wind structure on the sounds produced by meteors, PhD Thesis, Michigan Univ., Ann Arbor, MI, USA, 1974.

Rogers, A. E. E., Lekberg, M., and Pratap, P.: Seasonal and Diurnal Variations of Ozone near the Mesopause from Observationsof the 11.072-GHz Line, J. Atmos. Ocean. Tech., 26, 2192-2199, 2009.

Sarma, G. S. R.: Physico-chemical modelling in hypersonic flow simulation, Prog. Aerosp. Sci., 36, 281-349, 2000.

Smith, A. K. and Marsh, D. R.: Processes that account for the ozone maximum at the mesopause, J. Geophys. Res.-Atmos., 110, 11 pp., doi:10.1029/2005JD006298, 2005.

Smith, A. K., López-Puertas, M., Funke, B., García-Comas, M., Mlynczak, M. G., and Holt, L. A.: Nighttime ozone variability in the high latitude winter mesosphere, J. Geophys. Res.-Atmos., 119, 13547-13564, doi:10.1002/2014JD021987, 2015.

Sugar, G. R.: Radio propagation by reflection from meteor trails, Proceedings of the IEEE, 52, 116-136, doi:10.1109/PROC.1964.2801, 1964.

Sukara, R. E.: Potential for Measurement of Mesospheric Ozone Density from Overdense Meteor Trains with a Monostatic Meteor Radar, MSc Thesis, Dept. of Physics and Astronomy, Western University, London, Ontario, Canada, 2014.

Tabor, D.: Gases, liquids and solids, Penguin library of physical sciences: Physics/Chemistry, 290 pp., 1969.

Thomas, R. J.: Seasonal ozone variations in the upper mesosphere, J. Geophys. Res.-Atmos., 95, 7395-7401, doi:10.1029/JD095iD06p07395, 1990.

Tsikulin, M. A.: Shock waves during the movement of large meteorites in the atmosphere, Report No. NIC-Trans-3148, Naval Intelligence Command, Translation Div., Alexandria, VA, USA, 1970.

Vondrak, T., Plane, J. M. C., Broadley, S., and Janches, D.: A chemical model of meteoric ablation, Atmos. Chem. Phys., 8, 70157031, doi:10.5194/acp-8-7015-2008, 2008.

Weryk, R. J. and Brown, P. G.: Simultaneous radar and video meteors - I: Metric comparisons, Planet. Space Sci., 62, 132-152, doi:10.1016/j.pss.2011.12.023, 2012.

Weryk, R. J. and Brown, P. G.: Simultaneous radar and video meteors-II: Photometry and ionisation, Planet. Space Sci., 81, 32-47, doi:10.1016/j.pss.2013.03.012, 2013. 
Whalley, C. L. and Plane, M. C.: Meteoric ion layers in the Martian atmosphere, Faraday Discuss., 147, 349-368, 2010.

Whalley, C. L., Martín, J. C. G., Wright, T. G., and Plane, J. M.: A kinetic study of $\mathrm{Mg}+$ and $\mathrm{Mg}$-containing ions reacting with $\mathrm{O}_{3}$, $\mathrm{O}_{2}, \mathrm{~N}_{2}, \mathrm{CO}_{2}, \mathrm{~N}_{2} \mathrm{O}$ and $\mathrm{H}_{2} \mathrm{O}$ : implications for magnesium ion chemistry in the upper atmosphere, Phys. Chem. Chem. Phys., 13, 6352-6364, doi:10.1039/C0CP02637A, 2011.
Zel'dovich, Y. B. and Raizer, Y. P.: Physics of shock waves and high-temperature hydrodynamic phenomena, Dover Publications, Mineola, NY, USA, 2002. 